\title{
FGF-dependent metabolic control of vascular development
}

\author{
Pengchun $\mathrm{Yu}^{1}$, Kerstin Wilhelm ${ }^{\# 2}$, Alexandre Dubrac $\# 1$, Joe K. Tung ${ }^{\# 1}$, Tiago C. Alves ${ }^{3}$, \\ Jennifer S. Fang ${ }^{1}$, Yi Xie ${ }^{1}$, Jie Zhu ${ }^{4}$, Zehua Chen ${ }^{5}$, Frederik De Smet ${ }^{6,7}$, Jiasheng Zhang ${ }^{1}$, \\ Suk-Won Jin ${ }^{1,8}$, Lele Sun ${ }^{9}$, Hongye Sun ${ }^{9}$, Richard G. Kibbey ${ }^{3}$, Karen K. Hirschi ${ }^{1}$, Nissim \\ Hay $^{10}$, Peter Carmeliet ${ }^{11,12}$, Thomas W. Chittenden ${ }^{5}$, Anne Eichmann ${ }^{1,13}$, Michael Potente ${ }^{2}$, \\ and Michael Simons ${ }^{1,14}$ \\ ${ }^{1}$ Yale Cardiovascular Research Center, Yale University School of Medicine, New Haven, USA \\ ${ }^{2}$ Angiogenesis \& Metabolism Laboratory, Max Plank Institute for Heart and Lung Research, \\ D-61231 Bad Nauheim, Germany \\ ${ }^{3}$ Section of Endocrinology, Department of Internal Medicine, Yale University School of Medicine \\ ${ }^{4}$ Department of Cellular and Molecular Physiology, Yale University School of Medicine \\ ${ }^{5}$ Computational Statistics and Bioinformatics Group, Advanced Artificial Intelligence Research \\ Laboratory, WuXi NextCODE, Cambridge, MA, USA \\ ${ }^{6}$ Switch Laboratory, VIB-KU Leuven, Leuven, B-3000, Belgium \\ ${ }^{7}$ Center for Molecular Oncologic Pathology, Dana-Farber Cancer Institute, Boston, MA 02215, \\ USA
}

${ }^{8}$ School of Life Sciences, Gwangju Institute of Science and Technology, Gwangju, Korea

${ }^{9}$ Genomics Laboratory, WuXi NextCODE, Shanghai, China

${ }^{10}$ Department of Biochemistry and Molecular Genetics, College of Medicine, University of Illinois at Chicago

${ }^{11}$ Laboratory of Angiogenesis and Neurovascular Link, Department of Oncology, University of Leuven, Leuven, B-3000, Belgium

${ }^{12}$ Laboratory of Angiogenesis and Neurovascular Link, Vesalius Research Center, VIB, Leuven, B-3000, Belgium

13U970, Paris Cardiovascular Research Center, 56 Rue Leblanc, 75015 Paris, France

\footnotetext{
Users may view, print, copy, and download text and data-mine the content in such documents, for the purposes of academic research, subject always to the full Conditions of use:http://www.nature.com/authors/editorial_policies/license.html\#terms

Address correspondence to: Professor Michael Simons, Yale Cardiovascular Research Center, 300 George St, Rm 781, New Haven, CT 06511, USA. Phone: 203.737.4643; Fax: 203.737.5528; michael.simons@ yale.edu.

Author contributions

PY performed most of the experiments and prepared figures. KW analyzed Myc mutant mice and performed other Myc-related experiments. AD performed retina analysis and sprouting assays. JT carried out sprouting assay and Western blotting analysis. TA, JF, YX, FDS and JSZ performed mass spectrometry, FACS analysis, ChIP, SSR studies and cornea lymphangiogenesis assay respectively. JZ carried out Western blotting analysis. ZC, LS, HS and TC contributed to RNA-seq analysis. NH provided $H k 2^{\text {flox }} / f l o x$ mice. SJ, RK, KH, PC, AE and MP discussed results and provided comments. MS supervised the project and wrote the manuscript together with PY.

Competing financial interests: none.
} 
${ }^{14}$ Department of Cell Biology, Yale University School of Medicine, New Haven, CT

\# These authors contributed equally to this work.

\section{Abstract}

Blood and lymphatic vasculatures are intimately involved in tissue oxygenation and fluid homeostasis maintenance. Assembly of these vascular networks involves sprouting, migration and proliferation of endothelial cells. Recent studies have suggested that changes in cellular metabolism are of importance to these processes1. While much is known about vascular endothelial growth factor (VEGF)-dependent regulation of vascular development and metabolism2,3, little is understood about the role of fibroblast growth factors (FGFs) in this context4. Here we identify FGF receptor (FGFR) signaling as a critical regulator of vascular development. This is achieved by FGF-dependent control of c-MYC (MYC) expression that, in turn, regulates expression of the glycolytic enzyme hexokinase 2 (HK2). A decrease in HK2 levels in the absence of FGF signaling inputs results in decreased glycolysis leading to impaired endothelial cell proliferation and migration. Pan-endothelial- and lymphatic-specific $H k 2$ knockouts phenocopy blood and/or lymphatic vascular defects seen in Fgfr 1/r3 double mutant mice while HK2 overexpression partially rescues the defects caused by suppression of FGF signaling. Thus, FGF-dependent regulation of endothelial glycolysis is a pivotal process in developmental and adult vascular growth and development.

FGFR 1 is the most prominent $F G F R$ in both mouse and human lymphatic endothelial cells (LECs, Extended Data Fig. 1a, b). Knockdown of FGFR1 resulted in upregulation of FGFR3 expression while FGFR3 downregulation had no effect on other FGFR expression (Extended Data Fig. 1c, d). Cdh5-CreER 5 and Prox 1-CreER ${ }^{T 2(B A C)} 6$ driver lines were crossed with Fgfr $1^{\text {flox/flox }}$ and $\mathrm{Fgfr}^{-/-}$mouse lines to generate pan-endothelium $\left(\mathrm{Fgfr}^{\mathrm{iLEC}} ; \mathrm{Fgfr}^{-{ }^{-}-}\right)$and lymphatic endothelium $\left(F g f r 1^{i \Delta L E C(B A C)} ; F f r 3^{--}\right)$specific knockouts. The excision efficiency of both Cre drivers was assessed by crossing them with the $m T m G$ reporter mice. Cre activation at embryonic day (E)12.5 and E13.5 resulted in a high degree of recombination in the skin lymphatic vessels at E15.5 with both Cre deleters (Extended Data Fig. 1e, f).

The effect of these deletions on lymphatic development was examined using embryonic skin as a readout7. LECs start to invade anterior dorsal skin at E12.5 and migrate towards the dorsal midline. By E15.5-E16, lymphatic vessels from both sides fuse at the dorsal midline forming a primary lymphatic network (Fig. 1a, b). Whole-mount staining of the embryonic mouse skin with anti-VEGFR3 and PECAM1 antibodies in single knockout Fgfr $1^{i \Delta L E C(B A C)}$ or $\mathrm{Fgfr}^{-\leftarrow}$ mice revealed no abnormalities in lymphatic front migration (Extended Data Fig. 1g-j). Pan-endothelial Fgfrl deletion on the Fgfr.- ${ }^{-/}$background at E10.5 resulted in significant edema, the appearance of blood-filled lymphatics and reduced dermal lymphatic development (Extended Data Fig. 2a-e). When the deletion was activated a day later (E11.5), reduced migration and branching of lymphatics were still evident (Extended Data Fig. 2f-i). Analysis of LEC-specific Fgfr 1/r3 double knockout mice (tamoxifen treatment at E12.5 and E13.5) confirmed these findings, showing decreased LEC front migration, branching, and lower number of LECs in the skin (Fig. 1c-g). There was no appreciable difference in the 
size of the skin lymphatic vessels (Fig. 1f, h). To explore FGFR inhibition in suppression of pathological lymphangiogenesis, mice with orthotopic Panc02 tumors were orally treated with the SSR128129E (SSR) inhibitor8. There was a significant reduction of lymphangiogenesis in the peri-tumoral area in the inhibitor-treated mice compared to vehicle controls (Extended Data Fig. 2j, k), indicating a potential therapeutic value of FGFR inhibitors as anti-lymphangiogenic agents.

We next examined if FGF signaling plays a similar role in the blood vessel development. Analysis of $\mathrm{Fgfr}^{i \Delta E C} ; \mathrm{Fgfr}^{--}{ }^{--}$mice following Cre activation at E10.5 showed a significant reduction in vessel branching and coverage in the skin at E15.5 (Extended Data Fig. 3a-d). Examination of the arterial vasculature, revealed by Connexin 40 (Cx40) staining, also showed a reduction in branching (Extended Data Fig. 3f, g) but no differences in capillary or arterial diameter (Extended Data Fig. 3e, h).

The involvement of FGF signaling in blood vascular development was further confirmed by examining retinal vasculature in $F g f r 1^{i \Delta E C ;} ; F g f r^{-/-}$mice after Cre activation at postnatal day (P)0 (Extended Data Fig. 4a). There was a significant impairment of vascular growth and branching (Extended Data Fig. 4b, c), marked reduction in the number of tip cells (Extended Data Fig. 4d, e) and the extent of proliferation (Extended Data Fig. 4f, g). There is no

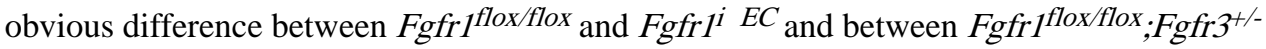
and $\mathrm{Fgfr} \mathrm{flox}^{\mathrm{fflox}} ; \mathrm{Fgfr} 3^{--}$in vascular density and branching (Extended Data Fig. 4h-k). A knockdown of FGFR1 in human dermal lymphatic endothelial cells (HDLECs) significantly reduced cell proliferation and migration while FGFR3 downregulation had no effect (Extended Data Fig. 5a-c). A double FGFR1/R3 knockdown effects were like those of FGFR1 knockdown (Extended Data Fig. 5a-c).

To establish the molecular basis of FGF-dependent regulation of vascular development, we carried out RNA sequencing (RNA-seq) analysis of LECs following stimulation with FGF2 or FGFR1 knockdown. Gene ontology analysis showed the expected statistical enrichment of molecular pathways related to cell proliferation and migration (Fig. 2a). Surprisingly, there also was enrichment among cellular metabolism processes and, especially, glucose metabolism pathways. To assess this aspect of FGF biology, we first examined contributions of major metabolic pathways to LEC energy generation. Flux analysis demonstrated that glycolysis was the most active process in LECs (Extended Data Fig. 6a), contributing to $>70 \%$ of the total ATP generation. HDLEC treatment with FGF2 doubled their glycolytic flux (Fig. 2b) and significantly increased glucose uptake (Extended Data Fig. 6b). Conversely, knockdown of FGFR1 reduced the flux rate (Fig. 2b). Steady-state levels of various glycolytic metabolites and lactate were all increased by FGF2 stimulation and decreased after FGFR1 knockdown (Fig. 2c, d). FGF signaling activation increased, while FGFR1 downregulation reduced, ATP production in HDLECs (Fig. 2e, f), consistent with the major contribution of glycolysis to energy generation.

To define the regulatory step involved in FGF-dependent control of LEC glycolysis, we analyzed the expression of rate-limiting glycolytic enzymes, including hexokinase (HK1 and HK2), phosphofructokinase (PFKP), and pyruvate kinase (PKM2). We also assessed 6phosphofructo-2-kinase/fructose-2,6-bisphosphatase 3 (PFKFB3) which regulates blood 
vessel sprouting9. HDLEC stimulation with FGF2 induced a robust increase in HK2 expression, with minimal expression changes of other enzymes (Fig. 2g, h) while FGFR1 knockdown led to a significant reduction in HK2 (Fig. 2i, j). Examination of skin LECs isolated from E15.5 Fgfr ${ }^{i \Delta L E C(B A C)}$; $F g f r 3^{--}$embryos confirmed the reduction in $\mathrm{Hk} 2$ expression (Fig. 2k). The importance of HK2 was supported by analysis of RNA-seq data: it was the only glucose metabolic gene among the top twenty transcripts (ranked by fold change) induced by FGF2 and downregulated by FGFR1 knockdown (Extended Data Fig. 6c).

HK2 knockdown significantly reduced, while adenoviral-mediated HK2 overexpression increased, the glycolytic flux (Extended Data Fig. 6d-f). Since the knockout of Fgfr 1/r3 in the endothelium also reduced angiogenesis, we examined whether FGF signaling regulates glycolysis and enzyme expression in blood endothelial cells (BECs). As in LECs, treatment of human umbilical vein endothelial cells (HUVECs) with FGF2 enhanced glycolysis and selectively induced HK2 expression (Extended Data Fig. 6g-i), indicating that FGF regulation of angiogenesis and lymphangiogenesis share similar metabolic mechanisms.

Besides FGF2, several other growth factors including VEGFC and insulin-like growth factors 1 and 2 (IGF1 and IGF2) can regulate lymphangiogenesis10. We tested if any of them also influences glycolysis in HDLECs. While all were effective in increasing cell proliferation, only VEGFC stimulation increased glycolytic flux, albeit to a lesser extent than FGF2 (Extended Data Fig. 6j). Consistent with these findings, only VEGFC increased HK2 expression without affecting other enzymes (Extended Data Fig. 6k).

FGF2 stimulation of HDLEC proliferation and migration was significantly reduced by HK2 knockdown (Extended Data Fig. 7a, b) while FGFR1 knockdown-induced decrease in HDLEC proliferation and migration was rescued by adenoviral HK2 expression (Extended Data Fig. 7c, d). FGF2-induced LEC sprouting was almost completely blocked by HK2 knockdown (Extended Data Fig. 7e). FGFR1 knockdown in HDLECs also fully blocked FGF2-induced sprouting while transduction of HK2 into HDLECs following FGFR1 knockdown partially restored sprouting (Extended Data Fig. 7f).

We next generated a mouse line with an endothelial-specific deletion of $H k 2\left(H k 2^{i \Delta E C}\right)$ by crossing $H k^{\text {flox } / f l o x}$ mice11 with $C d h 5-C r e E R^{T 2}$. When examined at E15.5 after E12.5 Cre activation, $H k 2^{i \Delta E C}$ embryos displayed lymphedema (Extended Data Fig. 8a, b) and a reduction in the extent of lymphatic vessel branching and migration towards the midline (Extended Data Fig. 8c-e), while blood vascular development was largely normal (Extended Data Fig. 8f, g). Analysis of cell cycle progression in LYVE1 ${ }^{+}$PECAM $1^{+}$LECs from the embryonic skin of $H k 2^{i \Delta E C}$ and littermate control mice demonstrated a higher proportion of G1 and smaller proportion of S phase cells in Hk2 deficient LECs (Extended Data Fig. 8h).

To confirm that these defects in lymphatic development were not secondary to any effects of $H k 2$ deletion in the blood endothelium, we crossed Hk $2^{\text {flox/flox }}$ mice with Prox 1$C r e E R^{T 2(B A C)}$. Immunostaining with anti-VEGFR3 antibody demonstrated a significant reduction in the skin lymphatic vessel development and branching at E15.5 after E12.5 Cre activation (Fig. 3a-c). 
To examine the role played by Hk2 in adult lymphangiogenesis, FGF2-containing pellets were implanted into corneas of Prox 1-CreER ${ }^{T 2(K I)} 12 ; H k 2^{\text {flox/flox }}$ (referred to as $H k 2^{i \Delta L E C(K I)}$ ) and control mice (Fig. 3d). The Cre recombinase, which was activated at the adult stage to bypass the early lymphatic defects caused by $H k 2$ knockout, was highly efficient in driving recombination in cornea lymphatics (Fig. 3e). While adult mice corneas are devoid of both lymphatic and blood vasculature13, FGF2 pellet implantation led to robust stimulation of lymphangiogenesis in control mice that was significantly reduced in $H k 2^{i \Delta L E C(K I)}$ mice (Fig. 3f, g). Similar to its role in the lymphatic development, panendothelial $H k 2$ deletion at early developmental stage (E10.5) significantly reduced angiogenesis (Extended Data Fig. 8i-1) as well as arterial development and branching (Extended Data Fig. 8m, n) in the embryonic mouse skin. As in the case of Fgfr1/r3, activation of endothelial $\mathrm{Hk} 2$ excision at $\mathrm{P} 0$ led to a significant reduction in the extent of development of the retinal vasculature (Extended Data Fig. 9a-c). The number of tip cells was reduced (Extended Data Fig. 9d, e) as was endothelial cell proliferation (Extended Data Fig. 9f, g). Vascular regression was not changed (Extended Data Fig. 9h, i).

Given RNA-seq demonstration of FGF-dependent regulation of $H K 2$ mRNA levels and a previous observation of MYC binding to the regulatory region of the $H K 2$ gene14, we examined whether MYC links FGF signaling to $H K 2$ transcription in HDLECs. Chromatin Immunoprecipitation (ChIP) confirmed MYC binding to conserved E-boxes in the first intron of the HK2 gene (Extended Data Fig. 10a, b). Moreover, a knockdown of MYC decreased, while its overexpression increased, HK2 mRNA levels (Extended Data Fig. 10c, d). MYC knockdown also reduced glycolysis (Extended Data Fig. 10e, f) while MYC overexpression increased glycolytic activity (Extended Data Fig. 10g).

FGF2 treatment of HDLECs increased MYC protein expression (Fig. 4a) while FGFR1 knockdown reduced it (Fig. 4b). Furthermore, ChIP-quantitative PCR (ChIP-qPCR) showed that the amount of MYC binding to the HK2 E-boxes was increased by FGF2 treatment and reduced by FGFR1 knockdown (Fig. 4c). MYC knockdown selectively reduced HK2 expression and prevented FGF2-induced increase in HK2 levels (Fig. 4d). Finally, the decrease in HK2 expression following FGFR 1 knockdown was completely rescued by overexpression of MYC (Fig. 4e).

Myc is highly enriched in embryonic dermal LECs (Extended Data Fig. 10h). Examination of the lymphatic vasculature in the anterior dorsal skin at E15.5 in Cdh5-

$\mathrm{CreER}^{T 2} ; \mathrm{Myc}^{\text {flox/flox }}\left(\mathrm{Myc}^{\mathrm{i} \Delta E C}\right)$ mice following Cre activation at E11.5 showed, similarly to Fgfr $1 / r 3$ and $H k 2$ knockout mice, the presence of edema (Fig. 4f) and a reduction in lymphatic vessel growth (Fig. 4g, h). LEC-specific Myc deletion using Prox1-CreER ${ }^{T 2(B A C)}$ confirmed these findings (Extended Data Fig. 10i-k).

In agreement with the study demonstrating Myc involvement in blood vasculature development15, MYC knockdown in HUVECs reduced HK2 mRNA and protein expression (Extended Data Fig. 101-n) while its overexpression increased HK2 levels (Extended Data Fig. 10o). Consistently, BECs isolated from $M y c^{i \Delta E C}$ embryos showed reduced expression of $H k 2$ but not $H k 1$ (Extended Data Fig. 10p). FGF2 treatment of HUVECs increased MYC expression (Extended Data Fig. 10q, r). Finally, FGF regulation of MYC was confirmed by 
the reduction of Myc expression in retinal vasculature of P5 $\mathrm{Fgfr}^{\mathrm{i \Delta EC}} ; \mathrm{Fgfr}^{-/}$mice (Extended Data Fig. 10s, t). Collectively, these results suggest that FGF-dependent regulation of MYC expression underlies control of HK2 levels in lymphatic and blood endothelial cells.

These data indicate that FGF signaling plays a pivotal role in both blood and lymphatic vascular development and it is also required for lymphangiogenesis in tumors. At the molecular level, FGFs control glycolysis via a MYC-dependent regulation of HK2 expression. FGF stimulation increased HK2 levels leading to induction of glycolysis and increased production of glycolytic metabolites, while its suppression had the opposite effect. MYC mediates FGF2 effects on HK2 expression by directly binding to $H K 2$ regulatory elements and controlling its transcription. Pan-endothelial and LEC-specific knockout of $M y c$ induces a phenotype closely resembling those seen in $\mathrm{Fgfr}^{i \Delta E C}$; Fgfr $3^{-/}$,

Fgfr $1^{i \Delta L E C(B A C)} ; F_{g f r} 3^{--}, H k 2^{i \Delta E C}$ and $H k 2^{i \Delta L E C(B A C)}$ mice. Thus, the FGF-MYC-HK2 axis is the crucial driver of glycolytic metabolism in the endothelium. The dominant role of HK2 in cellular metabolism appears to be restricted to cells predominantly relying on glycolysis (rather than oxidative metabolism) for energy generation. This agrees with the reported HK2 role in cancer metabolism (another highly glycolytic environment) 11.

The FGF/MYC/HK2-dependent regulation of vascular development is unexpected. Previously FGF activity has been linked to prevention of endothelium-to-mesenchymal transition both in the lymphatic16 and systemic vasculature17, injury response18, and maintenance of vascular integrity 19. While the FGFR1 and FGFR3 are the receptors involved, which of the 22 FGF family members is responsible for the required FGF signaling input is not known. In summary, FGF signaling regulates blood and lymphatic vascular development via control of endothelial metabolism driven by MYC-dependent regulation of HK2 expression. Therapeutic targeting of this FGF-MYC-HK2 pathway may open new possibilities for treatment of diseases associated with insufficient or excessive vascular growth.

\section{Methods}

\section{Genetically engineered mouse models}

For inducible Cre-mediated recombination, Fgfr $1^{\text {flox } / f l o x} ; \mathrm{Fgfr} 3^{+/-}$mice20 (mixed background), $H k 2^{\text {flox/flox }}$ mice11 (C57BL/6 background), and $M y c^{\text {flox/flox }}$ mice 21 (C57BL/6 background) were bred with $C d h 5-C r e E R^{T 2} 5$ (C57BL/6 background), Prox 1CreER ${ }^{T 2(B A C)} 6$ (mixed background) and Prox 1-CreER ${ }^{T 2(K I)} 12$ (mixed background).

$m T m G$ reporter mice22 (C57BL/6 background) were bred with different Cre lines to assess the recombination efficiency in lymphatics. All mouse protocols and experimental procedures were approved by the IACUC.

\section{Cell culture and treatment}

Human dermal lymphatic endothelial cells (HDLECs, HMVEC-dLyNeo-Der Lym Endo EGM-2MV) and pooled human umbilical vein endothelial cells (HUVECs) were purchased from Lonza and cultured in EBM2 basal medium with EGM-2 MV BulletKit. Both cell 
types were tested negative for mycoplasma in Lonza. Culture medium was changed every other day. Tissue culture plates were coated with $0.1 \%$ gelatin (Sigma) for $30 \mathrm{~min}$. at $37^{\circ} \mathrm{C}$ and washed with Dulbecco's Phosphate-Buffered Saline (Life Technologies) before cell plating. For siRNA-mediated gene knockdown, FGFR1 siRNA (ON-TARGETplus human FGFR1 siRNA, Dharmacon; SMARTpool siRNAs with four target sequences: GCCACACUCUGCACCGCUA, CCACAGAAUUGGAGGCUAC, CAAAUGCCCUUCCAGUGGG, GAAAUUGCAUGCAGUGCCG), FGFR3 siRNA (Hs_FGFR3_6, Qiagen; target sequence: CCGATGTTATTAGATGTTACA), HK2 siRNA (s6562 human HK2 siRNA, Life Technologies; sense sequence: CAGAGGUUCGAGAAAAUGAtt), c-MYC siRNA (ON-TARGETplus human Myc siRNA, Dharmacon; SMARTpool siRNAs with four target sequences: ACGGAACUCUUGUGCGUAA, GAACACACAACGUCUUGGA, AACGUUAGCUUCACCAACA, CGAUGUUGUUUCUGUGGAA), or AllStar negative control siRNA (Qiagen) was transfected by lipofectamine RNAimax reagent (Life Technologies). Human FGF2 was provided by Kaken Pharmaceutical Co, Ltd (Japan) or ordered from Peprotech. Human VEGFC, VEGFD, IGF1, IGF2 and PDGFBB were purchased from Peprotech. To assay the effect of FGFR1 knockdown on glycolytic enzyme expression, HDLECs, transfected with control or FGFR1 siRNA 3 days in advance, were replated and collected for protein analysis approximately $24 \mathrm{hr}$. later when the cell confluency reached $\sim 80 \%$. To examine the influence of growth factor treatment on glycolytic enzymes, freshly plated HDLECs were starved overnight with EBM2 plus $0.1 \%$ FBS and then stimulated with FGF2 (100 ng/ml or $200 \mathrm{ng} / \mathrm{ml})$, VEGFC (100 ng/ml), VEGFD (100 ng/ml), IGF1 (100 ng/ml), IGF2 (100 ng/ml) and PDGFBB (100 ng/ml) before lysis in RIPA buffer for protein extraction. For Western blot analysis the following antibodies were used: HK1 (Cell Signaling Technology, \#2024), HK2 (Cell Signaling Technology, \#2867), PFKFB3 (Proteintech, \#13763-1-AP), PFKP (Cell Signaling Technology, \#12746), PKM2 (Cell Signaling Technology, \#4053), c-MYC (Abcam, \#ab32072; Cell Signaling Technology, \#9402), FGFR1 (Cell Signaling Technology, \#9740), $\beta$-actin (Sigma, \#A5316), and Tubulin (Cell Signaling Technology, \#2148). ImageJ was used for densitometry quantification of western blot bands.

\section{RNA-seq experimental design and sample preparation}

HDLECs treated with or without FGF2 for $14 \mathrm{hr}$. were lyzed for RNA extraction and were eventually used to generate a list of differentially expressed genes between FGF2 and control, named "gain-of-FGF signaling" dataset. Similarly, HDLECs treated with FGFR1 siRNA or negative control siRNA for 3 days were lyzed for RNA extraction and were finally used to generate a list of differentially expressed genes between FGFR1 siRNA and negative control siRNA, so called "loss-of-FGF signaling" dataset. For each treatment, 9 replicates prepared from 3 independent experiments were analyzed. RNA isolation was carried out using RNeasy Mini Kit (Qiagen).

\section{RNA-seq}

Extracted total RNA was quantitated by NanoDrop and RIN value was measured with an Agilent Bioanalyzer. $1 \mu \mathrm{g}$ of qualified RNA (RIN >8.0) was used as input for library construction following the Illumina TruSeq RNA Sample Preparation protocol. RNA 
libraries were sequenced on an Illumina HiSeqX platform, PE 2x150bp. The average data yield for each sample was 20M PE reads with \% of Q30 bases $>90$.

\section{RNA-seq data analysis}

RNA-seq reads from each sample were aligned to human genome (build 38) using short reads aligner STAR (version 2.5.1b)23. Gene expression quantification was then performed using RSEM24 with GENCODE annotation (release 24: http://www.gencodegenes.org). Differential analysis was performed using edgeR25 to identify genes with significant expression changes between groups. Genes observed to change significantly (FDR $<1 \mathrm{x}$ $10^{-2}$ ) in their expression in both "gain-of-FGF signaling" dataset and "loss-of-FGF signaling" dataset were first identified (1999 transcripts). If a gene is truly regulated by FGF signaling, it should show opposite changing direction between "gain-of-FGF signaling" dataset and "loss-of-FGF signaling" dataset. Therefore, next-step analysis was focused on the 929 transcripts whose expression was upregulated by FGF2 treatment but decreased after FGFR1 knockdown, and the 828 transcripts whose expression was reduced by FGF2 stimulation but increased after FGFR1 knockdown. Those 1757 transcripts were used for functional enrichment analysis by running GOseq, an algorithm that controls gene length bias in next-generation sequence data26. nGOseq, a modified version of the nEASE algorithm27,28 which also controls for gene length bias, was used to assess functional enrichment of nested GOseq terms. Briefly, each enriched upper-level GOseq term was used for nested GOseq (nGOseq) analysis to identify statistically enriched nested GO terms driving upper-level functional enrichment of non-specific GOseq terms. For generating data in Extended Data Fig. 6c, the top 50 transcripts which were increased by FGF2 treatment (ranked by fold change) and the top 50 transcripts which were reduced after FGFR1 knockdown (ranked by fold change), among those 1999 transcripts mentioned above, were first identified. Comparing the two lists of top hits resulted in an overlap containing 24 protein-coding genes. Those 24 genes were further ranked by the sum of their absolute fold changes in FGF2 treatment and FGFR1 knockdown conditions. After this ranking, the top 20 genes were shown in Extended Data Fig. 6c.

\section{Measurement of glycolysis, glucose oxidation, glutamine oxidation, fatty acid oxidation and glucose uptake}

Glycolysis was measured as previously described9. Briefly, subconfluent HDLECs cultured in 12-well plates were incubated with $1 \mathrm{ml} /$ well EBM2 medium (containing appropriate amount of serum and supplement) with $80 \mu \mathrm{Ci} / \mathrm{mmol} 5-{ }^{3} \mathrm{H}$-glucose (Perkin Elmer) for 2-3 hr.. Then $0.8 \mathrm{ml} /$ well medium was transferred into glass vials with hanging wells and filter papers soaked with $\mathrm{H}_{2} \mathrm{O}$. After incubation in a cell culture incubator for at least 2 days to reach saturation, filter papers were taken out and the amount of evaporated ${ }^{3} \mathrm{H}_{2} \mathrm{O}$ was measured in a scintillation counter. Glucose oxidation, glutamine oxidation and fatty acid oxidation were measured essentially as reported9. For measurement of glucose uptake, HDLECs were incubated with 2-[1-14 C]- deoxy-D-glucose $(2.5 \mu \mathrm{Ci} / \mathrm{ml}$, Perkin Elmer) for 10 min. before PBS washing (at least 3 times to get rid of all radioactive medium) and then lyzed with $500 \mu \mathrm{L} 0.1 \mathrm{~N} \mathrm{NaOH} .400 \mu \mathrm{l} \mathrm{NaOH}$ cell lysate for each sample was transferred to scintillation vials containing scintillation liquid and measured. 


\section{Mass spectrometry analysis of metabolites}

HDLECs were quenched by a rapid wash with ice-cold PBS and then collected in $150 \mu$ of an ice-cold solution containing $20 \%$ methanol, $0.1 \%$ formic acid, $1 \mathrm{mM}$ phenylalanine, 3 $\mathrm{mM} \mathrm{NaF}$ and $100 \mu \mathrm{M}$ EDTA. ${ }^{2} \mathrm{H}_{4}$-Taurine $(10 \mu \mathrm{M}, \mathrm{CDN}$ Isotopes) was used as a loading control. All the samples were lyophilized and resuspended in $50 \mu \mathrm{L}$ of water prior the LCMS/MS analysis. Samples were injected onto a Cogent Diamond Hydride ${ }^{\mathrm{TM}}$ column ( $2.2 \mu \mathrm{m}$ particle size, $2.1 \mathrm{~mm} \times 10 \mathrm{~cm}$ ) at a flow rate of $0.5 \mathrm{~mL} / \mathrm{min}$. Glycolytic intermediates were eluted isocratically with a $95 \%$ aqueous $/ 5 \%$ organic solvent mixture. The aqueous solution contained $15 \mathrm{mM}$ ammonium formate. The organic solution contained $60 \%$ acetonitrile, $35 \%$ isopropyl alcohol and $15 \mathrm{mM}$ ammonium formate. Samples were ionized by electrospray into an ABSCIEX 5500 QTRAP equipped with a SelexION for differential mobility separation (DMS) and acquired using multiple reaction monitoring (MRM) in negative mode, as described previously29. DMS-based separation of fructose-6-phosphate from glucose-6-phosphate, as well as the separation of ATP, ADP and AMP nucleotides, was achieved using no modifier. Isopropyl alcohol was used as modifier for the DMS-based separation of the remaining glycolytic intermediates. Retention times were confirmed with known standards and peaks integrated using Multiquant (ABSCIEX) using the following MRM transition pairs $\left(\mathrm{Q}_{1} / \mathrm{Q}_{3}\right)$ : 506/159 for ATP, 426/79 for ADP, 346/79 for AMP, 259/97 for glucose-6-phosphate, 259/97 for fructose-6-phosphate, 339/97 for fructose-1,6bisphosphate, 169/97 for dihydroxyacetone phosphate (DHAP), 185/79 for 3phosphoglycerate (3PG), 185/79 for 2-phosphoglycerate (2PG), 167/79 for phosphoenolpyruvate (PEP), 89/89 for lactate and 124/80 for endogenous taurine. Endogenous taurine was used as internal control for cell density as previously described 30 .

\section{Seahorse assays}

Metabolic analyses in HDLECs were performed with the Seahorse XFe96 analyzer (Agilent Seahorse) according to the manufacturer's recommendations. In brief, siRNA-transfected or adenovirus-transduced HDLECs (40,000 cells per well of a 96-well plate) were seeded on fibronectin-coated XFe96 microplates. After $2 \mathrm{hr}$., cell culture medium was changed to a non-buffered assay medium and cells were maintained in a non- $\mathrm{CO}_{2}$ incubator for $1 \mathrm{hr}$.. The Glycolysis stress test kit (Agilent Seahorse) was used to monitor the extracellular acidification rate (ECAR) under various conditions. Three baseline recordings were made, followed by sequential injection of glucose $(10 \mathrm{mM})$, the mitochondrial / ATP synthase inhibitor oligomycin $(3 \mu \mathrm{M})$, and the glycolysis inhibitor 2-deoxy-D-glucose (2-DG; 100 $\mathrm{mM})$.

\section{Quantitative PCR (qPCR) analysis}

RNA was extracted from cells using the RNeasy Mini Kit or the RNeasy Plus Mini Kit (Qiagen) according to the manufacturer's instructions. cDNA synthesis was performed using the M-MLV reverse transcriptase (Invitrogen) or the iScript cDNA synthesis kit (Bio-rad). qPCR was performed either with TaqMan Gene Expression Master Mix (Thermo Fisher Scientific) and TaqMan probes (Thermo Fisher Scientific), or with iQ ${ }^{\mathrm{TM}}$ SYBR Green Supermix (Bio-rad). For TaqMan method, the following assays were used: human $A C T B$ Hs99999903_m1; human $c$-MYCHs00153408_m1; human HK2 Hs00606086_m1; mouse 
Actb Mm02619580_g1; mouse Hk1 Mm00439344_m1; mouse Hk2 Mm00443385_m1. For SYBR method, qPCR primers for human FGFR1-FGFR4, human GAPDH, human $\beta$ ACTIN, mouse Fgfr 1-Fgfr 4 , mouse $H k 1$ and mouse $\beta$-Actin were ordered from Qiagen. Mouse $H k 2$ qPCR primers both purchased from Qiagen and designed in-house were used to generate data for Fig. 2k. The sequences of in-house designed qPCR primers are (5' to 3'): Mouse $H k 2$ (CGGTACACTCAATGACATCCGA; TTCACCAGGATGAGTCTGACC) and human RPLPO (TCTGCATTCTCGCTTCCTGG; CAGGACTCGTTTGTACCCGT).

\section{ChIP-qPCR}

ChIP assays were performed using SimpleChIP ${ }^{\circledR}$ Plus Enzymatic Chromatin IP Kit (Cell Signaling) according to manufacturer's protocol with some minor modifications. Cells cultured on $20-\mathrm{cm}$ dishes were fixed for $10 \mathrm{~min}$. by adding $37 \%$ formaldehyde solution to the culture medium to a final concentration of $1 \%$. Fixation was quenched with glycine for 5 $\mathrm{min}$. at room temperature. Cells were washed twice with ice-cold PBS, scraped into $2 \mathrm{ml}$ PBS, and centrifuged at 5,000 rpm for $10 \mathrm{~min}$.. The cell pellets from two 20-cm dishes were combined and lysed in $1 \mathrm{ml}$ lysis buffer. The lysate was then centrifuged at 5,000 rpm for 5 min. at $4^{\circ} \mathrm{C}$ and the pellet was resuspended in $100 \mu \mathrm{l}$ nuclease digestion buffer. The DNA was digested with $0.5 \mu \mathrm{l}$ of micrococcal nuclease for $20 \mathrm{~min}$. at $37^{\circ} \mathrm{C}$ to a length of approximately 150-900 bp (checked by agarose gel electrophoresis). Lysates were centrifuged and the pellet was resuspended in $500 \mu \mathrm{l} \mathrm{ChIP} \mathrm{buffer}$ and sonicated for $3 \times 30 \mathrm{~s}$ at power level 2 and $40 \%$ constancy. The solution was centrifuged at 10,000 rpm for 10 min., and the supernatant was collected which was the cross-linked chromatin. For chromatin immunoprecipitation (IP), $150 \mu \mathrm{l}$ of cross-linked chromatin was used for each IP and mixed with rabbit anti-c-MYC antibody (Abcam, 1: 50) or same amount of rabbit IgG control at $4{ }^{\circ} \mathrm{C}$ overnight. $2 \%$ of cross-linked chromatin was saved as input control for qPCR reaction later on. $30 \mu \mathrm{l}$ of Protein G magnetic bead slurry was added to each IP reaction and incubate for $2 \mathrm{hr}$. at $4{ }^{\circ} \mathrm{C}$ with rotation. The magnetic beads were washed 3 times with ChIP low salt buffer and once with ChIP high salt buffer. The bound chromatin on the beads was released in ChIP elution buffer by heating at $65{ }^{\circ} \mathrm{C}$ for $30 \mathrm{~min}$. with vortex at 1,200 rpm. The chromatin was then digested with Protease $\mathrm{K}$ and purified using spin column. The DNA was eventually eluted in $50 \mu \mathrm{l}$ DNA elution buffer. The amount of precipitated DNA from each sample was quantified by qPCR using primers flanking the MYC binding element in the $H K 2$ gene. The reading was normalized to that of DNA purified from the previously saved cross-linked chromatin (2\% input). The reading by DNA from IP using MYC antibody against that from IgG IP indicated the antibody efficiency for ChIP assay. The qPCR primers (flanking the E-boxes) for detecting the MYC binding element14 are (5'-3'): GCCCCGCAGGTAGTCAGG; AGCCACGATTCTCTCCACG.

\section{xCELLigence Real-Time Cell Analysis (RTCA)}

HDLEC proliferation was measured through using xCELLigence RTCA instrument (Roche Dignostics) and E-plate 16 (a modified 16-well plate, Roche Dignostics). E-plate 16 was coated with $0.1 \%$ gelatin, loaded with $100 \mu \mathrm{l}$ cell-free medium and left in tissue culture hood for $30 \mathrm{~min}$. to reach equilibrium. E-plate 16 was placed into RTCA instrument to measure the background impedance. Thereafter, $100 \mu \mathrm{l}$ cell suspensions with less than 8000 cells were added into each well of E-plate 16, which was then placed in tissue culture incubators 
for $30 \mathrm{~min}$. to allow cells to settle down before being measured by RTCA device. The impedance value of E-plate 16 was automatically monitored every $15 \mathrm{~min}$. with 3-4 replicates for each treatment. For experiments requiring measurement of FGF2 effect on cell proliferation, HDLECs were resuspended in EBM2 plus 3\% FBS before being plated into Eplate 16. 10-12 hr. after RTCA measurement, the monitor program was paused and E-plate 16 was taken out from the device to add $2 \mu \mathrm{FGF} 2$ or vehicle to each well, after which the measurement continued. For the other experiments, fully supplemented medium (EBM2 plus 5\% FBS and growth factors) was used in E-plate 16. The final data were presented as a plot of time versus normalized cell index (normalized to data at a time point of interest).

\section{Wound healing migration assay}

HDLEC migration was measured in a wound healing assay, which employed Ibidi cultureinserts (Ibidi) to generate the wound. An Ibidi culture-insert is $9 \mathrm{~mm} \times 9 \mathrm{~mm} \times 5 \mathrm{~mm}$ (w x 1 $\mathrm{x} \mathrm{h}$ ) and is composed of two wells. One or two inserts were placed into one well of 6-well plates. After being coated with $0.1 \%$ gelatin, both wells of inserts were loaded with $100 \mu \mathrm{l}$ cell suspension. When cells became fully confluent after attachment, culture inserts were carefully removed by sterile tweezers to start cell migration. For studying the effect of HK2 siRNA on FGF2-stimulated migration (FGF2 was ordered from R\&D Systems) and the effect of FGFR1 siRNA and/or FGFR3 siRNA on cell migration, would healing process was monitored for approximately $12 \mathrm{hr}$.. To assess the rescue effect of HK2 overexpression in FGFR1 siRNA-treated cells, cell migration was evaluated in approximately $17 \mathrm{hr}$.. Nikon ELIPSE TS100 microscope with a PixeLINK camera was used to image cells at the first time point $\left(\mathrm{T}_{0}\right)$ and the last time point $\left(\mathrm{T}_{\text {end point }}\right)$. For data analysis, ImageJ was used to measure the wound area in $\mathrm{T}_{0}$ and $\mathrm{T}_{\text {end point }}$. Migration area was obtained by subtracting Area $\left(T_{\text {end point }}\right)$ from Area $\left(T_{0}\right)$.

\section{Three-dimensional bead sprouting assay}

HDLECs were trypsinized and mixed with collagen-coated Cytodex ${ }^{\circledR}$ microcarrier beads (Sigma) in a ratio of 2,500 beads to $1 \times 10^{6}$ cells in warm EGM-2 medium in a $15 \mathrm{ml}$ falcon tube. The mixture was incubated at $37^{\circ} \mathrm{C}$ for $4 \mathrm{hr}$., with shaking every $20 \mathrm{~min}$. to ensure even coating of the beads. After $4 \mathrm{hr}$., the coated beads were transferred to a 6-well plate in $2 \mathrm{ml}$ of EGM-2 medium per well and incubated at $37^{\circ} \mathrm{C}$ overnight. The next day, coated beads

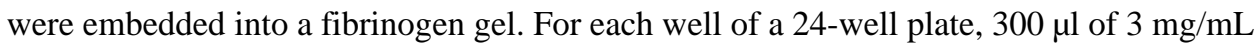
fibrinogen in PBS was used, along with $100 \mu \mathrm{g} / \mathrm{ml}$ of aprotinin (Sigma) and $1.5 \mathrm{unit} / \mathrm{ml}$ of thrombin (Sigma). Approximately 250 coated beads were embedded in each well. The plate was then incubated at $37^{\circ} \mathrm{C}$ for $1 \mathrm{hr}$. to generate a clot. After the gel solidified, human lung fibroblasts in EGM-2 medium were seeded on top at a concentration of 20,000 cells/well. The medium was changed every other day (full EGM-2 medium with $200 \mathrm{ng} / \mathrm{mL}$ of FGF2), and the plates were imaged on day 6 using a spinning disk confocal microscope (Perkin Elmer). ImageJ was used to measure the sprout length for the data analysis.

\section{Adenovirus}

Adenovirus encoding GFP, empty CMV vector, HK231, or MYC (from Vector Biolabs) for in vitro experiments was amplified in 293A cells according to the user manual of 
ViraPower ${ }^{\mathrm{TM}}$ Adenoviral Expression System (Life Technologies). Virus was tittered using Adeno- $\mathrm{X}^{\mathrm{TM}}$ Rapid Titer Kit (Clontech Laboratories).

\section{Analysis of lymphatic development}

To induce Cre activity during embryonic stage, each pregnant mouse was injected i.p. with 2 mg tamoxifen (Sigma, T5648) for two consecutive days (E10.5-E11.5, E11.5-E12.5 and E12.5-E13.5). Skin tissues were harvested at E15.5. Standard whole-mount immunohistochemistry procedure was carried out to stain the skin with antibodies against PECAM1 (BD Pharmigen, \#553370), VEGFR3 (R\&D Systems, \#AF743), PROX1 (Angiobio, \#11-002), Cx40 (ALPHA DIAGNOSTIC, \#CX40-A), EGFP (Life Technologies, \# A-11122; Abcam, \#ab13970) and Alexa fluorescent secondary antibodies (Life Technologies). A spinning disk confocal microscope (Perkin Elmer) and a Leica SP5 confocal microscope were used to generate high-resolution images of immunostained samples. Stitch imaging mode was chosen to image samples of large size. ImageJ was employed to crop representative area from large, stitched images for exhibition. For quantification of lymphatic development in the anterior dorsal skin, comparable regions (based on the blood vessel pattern) between different samples were selected and cropped out of those stitched images as regions of interest (ROIs) for further analysis. Lymphatic development parameters, e.g. branching points and LEC nucleus numbers, were measured using ImageJ with "Lymphatic Vessel Analysis" plugin. If considerably big area within a ROI was destroyed during skin dissection, that sample was excluded for analysis.

\section{Retinal vasculature analysis}

Gene deletion was induced by intragastric injections to pups with $50 \mu \mathrm{g}$ tamoxifen (1 $\mathrm{mg} / \mathrm{ml}$ ) at P0, P1 and P2. Mice were sacrificed at P5 for analysis of retinal vasculature as previously described32. The retinas were incubated with isolectin B4 (IB4) and the following antibodies: anti-Collagen IV (Millipore, \#AB769), anti-Erg1/2/3 (Santa Cruz, \#SC353), anti-Phospho-Histone H3 (PH3, Millipore, \#06-570). Retinas were imaged using a Nikon 80i fluorescence microscope and a Leica SP5 confocal microscope with a Leica spectral detection system (Leica 15 SP detector) and the Leica application suite advanced fluorescence (LAS-AF) software. Quantification of retinal vascular development and immunostaining were done using the Biologic CMM Analyser Software and ImageJ.

\section{Analysis of cell cycle distribution}

Dorsal skin explants were harvested from E15.5 mouse embryos, and minced into ice-cold Dulbecco's Modified Eagle's Medium supplemented with 20\% fetal bovine serum and 1.25 $\mathrm{mg} / \mathrm{ml}$ collagenase. Samples were incubated for $30 \mathrm{~min}$. at $37^{\circ} \mathrm{C}$, and mechanically dissociated by repeated pipetting until a single cell suspension was achieved. Samples were then centrifuged for $1 \mathrm{~min}$. at 2,000 $\mathrm{g}$ at $4^{\circ} \mathrm{C}$, and cell pellet was resuspended in PBS supplemented with $10 \%$ FBS, $5.5 \mathrm{mM}$ glucose, and $20 \mathrm{mM}$ HEPES. Cells were incubated in the presence of Hoechst $33342(25 \mu \mathrm{g} / \mathrm{ml}) 30 \mathrm{~min}$. at $37^{\circ} \mathrm{C}$, and then additionally for $15 \mathrm{~min}$. at $37^{\circ} \mathrm{C}$ in the presence of Pyronin $\mathrm{Y}(0.5 \mu \mathrm{g} / \mathrm{ml})$ as well as fluorescently conjugated antibodies: PECAM1-FITC (BD Pharmingen, \#553372) and LYVE1-Alexa647 (eBioscience, \#50-0443-82). Samples were washed and resuspended in $0.5 \mathrm{ml}$ PBS on ice for subsequent analysis. BECs and LECs were identified by flow cytometry as 
PECAM $1^{+}{ }^{L Y V E} 1^{-}$and PECAM1 ${ }^{+} \mathrm{LYVE} 1^{+}$events respectively, and for each population, cell cycle distribution was determined by relative DNA (Hoechst) and RNA (Pyronin Y) content.

\section{Fluorescence-activated cell sorting (FACS) to sort LECs for qPCR analysis}

Dorsal skin explants were harvested from E15.5 mouse embryos into ice-cold Dulbecco's Modified Eagle's Medium supplemented with $20 \%$ fetal bovine serum and $1 \mathrm{mg} / \mathrm{mL}$ collagenase. Samples were incubated for $1 \mathrm{hr}$. at $37^{\circ} \mathrm{C}$, and mechanically dissociated by repeated pipetting until a single cell suspension was achieved. Samples were then centrifuged for $1 \mathrm{~min}$. at 2,000 g at $4^{\circ} \mathrm{C}$, and the cell pellet was resuspended in Hank's Buffered Saline Solution supplemented with 10\% FBS, $5.5 \mathrm{mM}$ glucose, and $20 \mathrm{mM}$ HEPES. Cells were incubated in the presence of fluorescently conjugated antibodies PECAM1-FITC (BD Pharmingen, \#553372), CD45-PECy7 (eBioscience, \#25-0451-82) and LYVE1-Alexa647 (eBioscience, \#50-0443-82) for $30 \mathrm{~min}$. at $37^{\circ} \mathrm{C}$. Samples were pelleted for $1 \mathrm{~min}$. at 2,000 g at $4^{\circ} \mathrm{C}$, resuspended in $0.5 \mathrm{~mL}$ PBS on ice, and filtered through a $35 \mu \mathrm{m}$ nylon mesh prior to flow cytometry analysis and cell sorting. BECs were identified by FACS as PECAM $1^{+} \mathrm{CD} 45^{-} \mathrm{LYVE}^{-}$and LECs were identified as PECAM1 ${ }^{+} \mathrm{CD} 45^{-} \mathrm{LYVE} 1^{+}$events, and cells from each population were sorted into RLT lysis buffer (Qiagen) for mRNA preparation (RNeasy Micro kit), cDNA library construction, and subsequent qPCR analysis. When analyzing qPCR results, wells where sample melting curve peaks at the incorrect temperature were excluded.

\section{Corneal lymphangiogenesis model}

Slow-releasing pellets containing FGF2 were made as previously described33. Surgery to implant the pellets into the mouse cornea was performed as reported13. Adult Prox 1$C r e E R^{T 2(K I)} ; H k 2^{\text {flox/flox }}$ and control female mice were i.p. injected with tamoxifen (150 $\mu \mathrm{g} / \mathrm{g}$ body weight) every other day (seven injections in total) before the cornea surgery. 1 week after the pellet implantation, eyeballs were harvested for cornea dissection and immunostaining with LYVE1 (Angiobio, \#11-034) and PECAM1 (BD Pharmigen, \#553370) antibodies and then used for imaging (spinning disk confocal microscopy) and quantification analysis (ImageJ).

\section{Murine orthotopic pancreatic tumor model}

The murine pancreatic tumor cell line Panc02 was obtained from Prof. Wiedenmann (Charité University Hospital, Berlin). 1 x $10^{6}$ tumor cells were injected subcapsularly in the head region of the pancreas of anaesthetized female C57B1/6 mice (7-9 weeks old, Charles River, France) using a 30-gauge needle. At day 3 after tumor inoculation, tumor-bearing mice were treated daily with SSR (30 mg/kg/day) or vehicle ( $0.6 \%$ methylcellulose) via oral gavage. At day 9 , primary tumors were removed, weighted, and tumor volumes $(\mathrm{V})$ were calculated using the formula $\mathrm{V}=0.52 \times\left(\mathrm{a}^{2} \mathrm{x} b\right)$, where a represents the smallest tumor diameter and $b$ represents the largest tumor diameter. The incidence of tumor invasion into adjacent organs, hemorrhagic ascites, and regional celiac and mesenteric lymph node metastases were recorded, and confirmed by immunohistochemistry on paraffin sections. Paraffin-embedded $7 \mu \mathrm{m}$ sections were prepared and used for immunohistochemistry using the following antibodies: anti-LYVE1 (Upstate-Cell Signaling Solutions, Bio-connect, 
Huissen, The Netherlands) and anti-VEGFR3 (eBiosciences). The lymph vessel area in the peritumoral area was analysed using the KS300 software (Zeiss). Maximum tumor size (for orthotopic pancreatic tumor model) allowed by IACUC of the KU Leuven is $1.7 \mathrm{~cm}$. This limit was not exceeded in any of the experiments. Experiments were discontinued and mice were euthanised if the tumor reached either $>1.7 \mathrm{~cm}$ in any linear direction or a total volume of $2,000 \mathrm{~mm}^{3}$, if mice were not moving easily and spontaneously, if they were losing $\geq 20 \%$ of their body weight, or if they were clearly not comforted by the degree of analgesia.

\section{Statistical analysis}

No statistical analysis was performed to pre-determine sample size. For cornea lymphangiogenesis assay, the sample size was estimated based on a previous report13. Randomization and blinding was not used in our animal studies. Statistical analysis (statistical significance calculation and F test) was performed using GraphPad Prism 7. Statistical significance between two groups was determined by two-tailed unpaired t-test (assume normal distribution), and statistical significance between multiple groups was calculated using one-way ANOVA with post-hoc tests. Data represent mean value \pm standard error of the mean (s.e.m.).

\section{Data availability}

RNA-seq data are available at the Sequence Read Archive under accession SRP099111. All other data are available from the corresponding author upon reasonable request. 


\section{Extended Data}

a
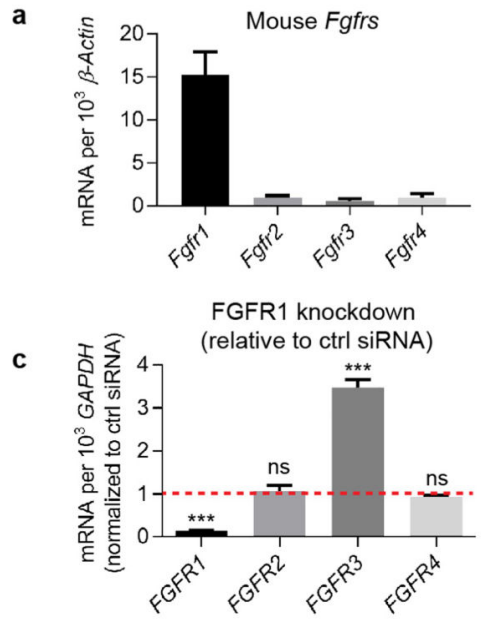

e

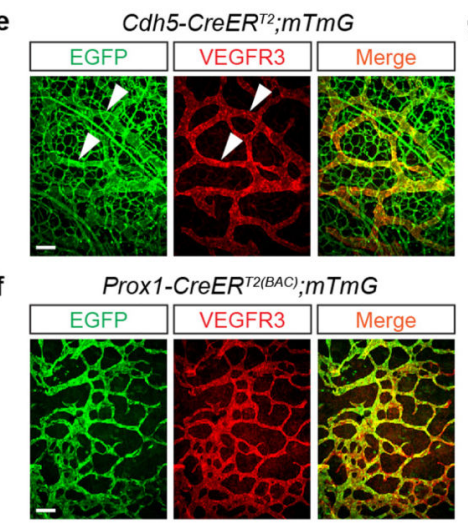

g b

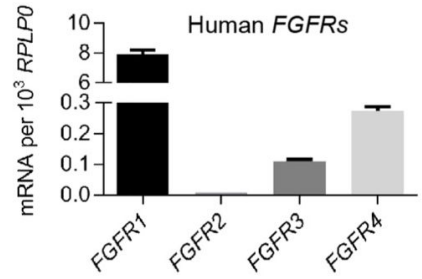

d

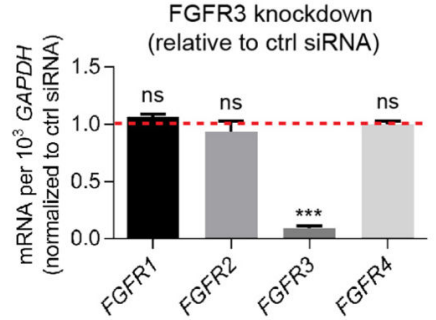

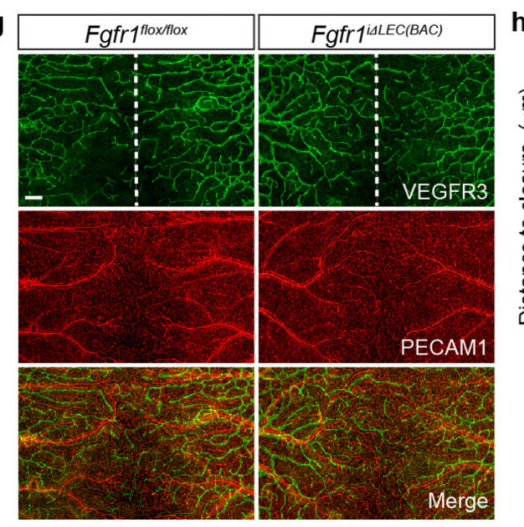

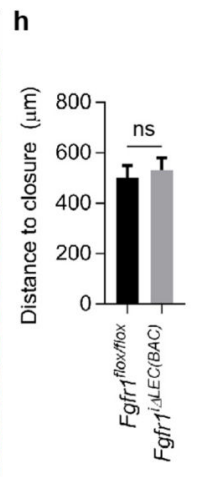

i
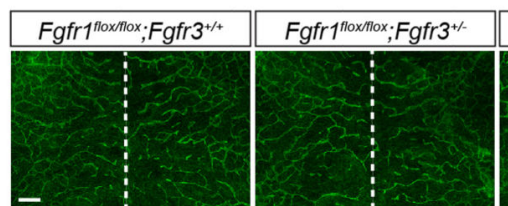

Fgfrt floxhlox: Fgfr $3^{-\%}$
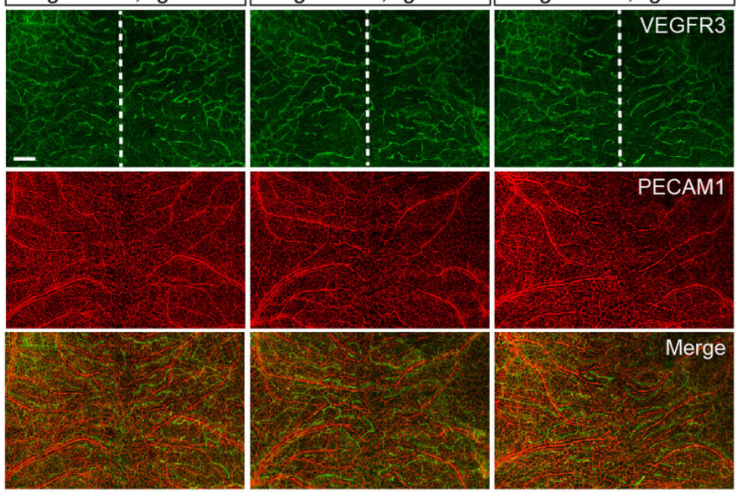

\section{j}

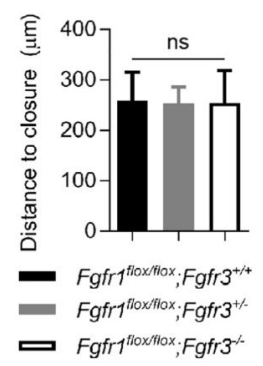

Extended Data Figure 1. Expression of FGFRs in mouse and human LECs and the effect of single knockout of $F g f r l$ or $F g f r 3$ on lymphatic development in the embryonic skin.

a, b, qPCR analysis of FGFR expression in mouse dermal LECs (isolated from E15.5 embryos by FACS) (a; $n=3$ embryos) and HDLECs (b; $n=3$ technical replicates, representative of 2 experiments). $\mathbf{c}, \mathbf{d}$, qPCR analysis of FGFR expression in HDLECs with FGFR1 (c) or FGFR3 (d) knockdown. FGFR mRNA levels in FGFR1 or FGFR3 deficient cells were presented as values relative to those of control siRNA-treated cells. $n=6$ 
replicates ( 2 independent experiments, technical triplicates per experiment). e, f, Activation of $m T m G$ reporter by $C d h 5-C r e E R^{T 2}(\mathbf{e}$; scale bar, $100 \mu \mathrm{m})$ or Prox 1-CreER ${ }^{T 2(B A C)}$ (f; scale bar, $250 \mu \mathrm{m}$ ) in dermal LECs of E15.5 mouse embryos. g, Anterior dorsal skin with VEGFR3 and PECAM1 staining from E15.5 Fgfr $1^{i \Delta L E C(B A C)}$ and $F g f r 1^{\text {flox/flox }}$ embryos, which were treated with tamoxifen at E12.5 and E13.5. Scale bar, $250 \mu \mathrm{m}$. h, Quantification of the distance between the two leading fronts of the lymphatic vessels $(n=6$ embryos for Fgfr Iflox/flox; $n=5$ embryos for Fgfr $1^{i \Delta L E C(B A C)}$ ). i, Anterior dorsal skin stained for VEGFR3 and PECAM1 from E15.5 Fgfr flox/flox $^{\text {Fgfr }} 3^{+/+}, \mathrm{Fgfr}^{\text {flox } / \text { flox }} ; \mathrm{Fgfr} 3^{+/}$, and Fgfr flox/flox Fgfr $3^{-/}$embryos. Scale bar, $250 \mu \mathrm{m}$. j, Quantification of the distance between the two leading fronts of the lymphatic vessels ( $n=4$ embryos for $F g f r 1^{\text {flox/flox }} ; \mathrm{Fgfr}^{+/+t} ; n=$

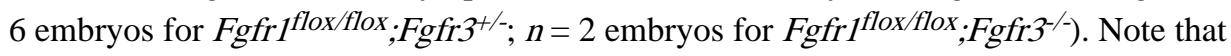
these embryos were not treated with tamoxifen. Dotted lines indicate the midline in $\mathbf{g}$ and $\mathbf{i}$. Data represent mean \pm s.e.m., $* * * P<0.001, \mathrm{~ns}=$ non-significant, calculated by unpaired $\mathrm{t}$ test $(\mathbf{c}, \mathbf{d}, \mathbf{h})$ and One-way ANOVA plus Tukey's multiple comparisons test $(\mathbf{j})$. 

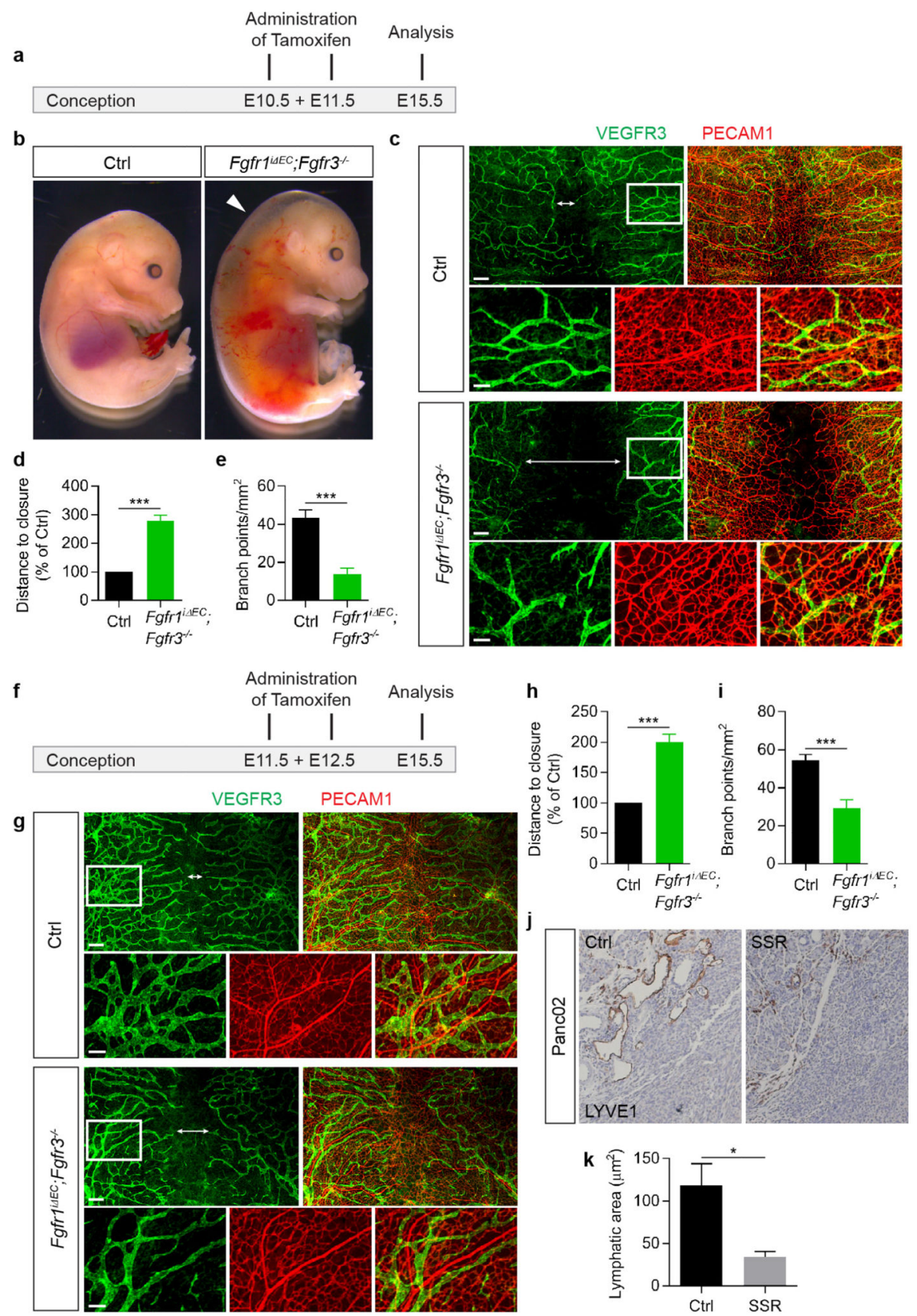

Extended Data Figure 2. Endothelial Fgfr1/r3 deletion impairs dermal lymphatic development and inhibition of FGF signaling suppresses pathological lymphangiogenesis.

a, Experimental strategy. b, Bright-field images of E15.5 Fgfr $1^{i \Delta E C} ; \mathrm{Fgfr}^{-/-}$and control (Fgfr ${ }^{\text {flox/flox }} ; \mathrm{Fgfr}^{+/-}$) embryos with tamoxifen treatment at E10.5 and E11.5. Arrowhead denotes area with lymphedema. c, Representative images of anterior dorsal skin with VEGFR3 and PECAM1 staining from E15.5 Fgfr $1^{i \Delta E C} ; \mathrm{Fgfr}^{-/-}$and control embryos treated with tamoxifen at E10.5 and E11.5. d, e, Quantification of the distance between the two leading fronts of the lymphatic vessels (d; $n=3$ litters) and the number of lymphatic branch 
points per $\mathrm{mm}^{2}$ skin area $\left(\mathbf{e} ; n=9\right.$ embryos for control $\left(\right.$ Fgfr $\left.1^{\text {flox } / \text { flox }} ; \mathrm{Fgfr}^{+/ /} \mathrm{C}^{+}\right) ; n=7$ embryos for $\left.\mathrm{Fgfr}^{i \Delta E C} ; \mathrm{Fgfr}^{--}{ }^{--}\right)$. f, Experimental strategy. $\mathbf{g}$, Representative images of anterior dorsal skin with VEGFR3 and PECAM1 staining from E15.5 Fgfr ${ }^{i \Delta E C} ; \mathrm{Fgfr}^{-3^{--}}$and control embryos with tamoxifen treatment at E11.5 and E12.5. h, i, Quantification of the distance between the two leading fronts of the lymphatic vessels (h; $n=4$ litters) and the number of lymphatic branch points per $\mathrm{mm}^{2}$ skin area (i; $n=9$ embryos for control $\left(\right.$ Fgfr $\left.1^{\text {flox } / \text { flox }} ; \mathrm{Fgfr} 3^{+-}\right) ; n=8$ embryos for $F g f r 1^{i \Delta E C} ; F_{g f r} 3^{--}$). In $\mathbf{c}, \mathbf{g}$, bottom panels (scale bar, $100 \mu \mathrm{m}$ ) for each genotype are high-magnification images of boxed regions in upper panels (scale bar, $250 \mu \mathrm{m}$ ). Double-headed arrows indicate the distance between the two

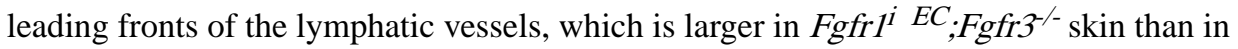
control. j, Representative images of the peritumoral area of orthotopic Panc02 tumors stained for LYVE1 following vehicle (control) or FGFR-inhibitor treatment (SSR). $\mathbf{k}$, Quantification of the area of LYVE1 ${ }^{+}$lymphatics per peritumoral area $(n=3$ mice for each condition). Data represent mean \pm s.e.m., $* P<0.05$, *** $P<0.001$, calculated by unpaired $\mathrm{t}$ test $(\mathbf{d}, \mathbf{e}, \mathbf{h}, \mathbf{i}, \mathbf{k})$. 

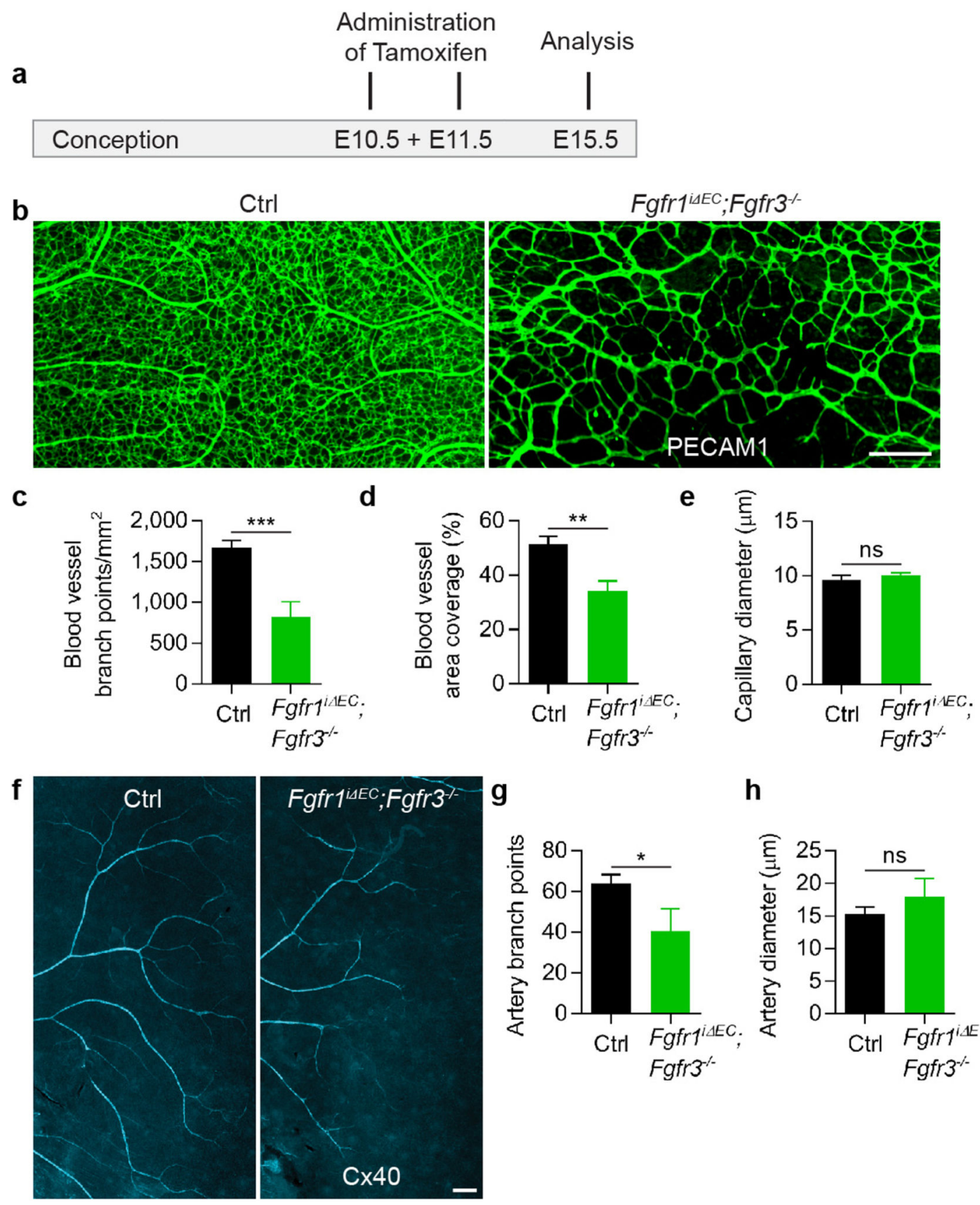

Extended Data Figure 3. Endothelial Fgfr1/r3 are essential for blood vessel development in the embryonic skin.

a, Schematic of the experimental strategy. $\mathbf{b}$, Representative images of anterior dorsal skin with PECAM1 staining from E15.5 Fgfr $1^{i \Delta E C} ; \mathrm{Fgfr}^{-/-}$and control embryos. Scale bar, 250 $\mu \mathrm{m}$. c-e, Quantification of the number of blood vessel branch points per $\mathrm{mm}^{2}$ skin area (c; $n$ $=9$ embryos for control ( Fgfr $\left.1^{\text {flox/flox }} ; \mathrm{Fgfr} 3^{+/-}\right) ; n=7$ embryos for $\left.F g f r 1^{i \Delta E C} ; F f_{r} 3^{-/-}\right)$, blood vessel covered area relative to skin area $(\mathbf{d} ; n=9$ embryos for control $\left(\right.$ Fgfr $\left.1^{\text {flox } / f l o x} ; \mathrm{Fgfr}^{+/}{ }^{+-}\right) ; n=7$ embryos for $\left.F g f r 1^{i \Delta E C} ; \mathrm{Fgfr}^{-/-}\right)$, and capillary diameter $(\mathbf{e} ; n=$ 
6 embryos for control ( Fgfr $\left.1^{\text {flox/flox }} ; F_{g f r} 3^{+/}\right) ; n=3$ embryos for Fgfr $\left.^{i \Delta E C} ; F^{i \Delta f r} 3^{--}\right)$. f,

Anterior dorsal skin stained for $\mathrm{Cx} 40$ in E15.5 Fgfr $1^{i \Delta E C} ; \mathrm{Fgfr}^{-1-}$ and control embryos. Scale bar, $250 \mu \mathrm{m} . \mathbf{g}, \mathbf{h}$, Quantification of the number of artery branch points $(\mathbf{g})$ and artery diameter (h). $n=6$ embryos for control ( Fgfr $\left.1^{\text {flox/flox }} ; \mathrm{Fgfr}^{+/-}\right) ; n=3$ embryos for

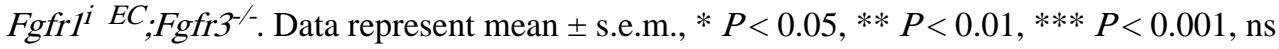
$=$ non-significant, calculated by unpaired t test $(\mathbf{c}-\mathbf{e}, \mathbf{g}, \mathbf{h})$.

Left panel

Vascular progression (d/D)

Ctrl

0.784435

0.67544

0.705641

0.725372

0.746389

0.741654

0.522593

0.579202

0.65251

0.645632

0.49403

0.57487

0.670957

0.645261

0.718848

0.75111

$F_{f r}{ }^{i \Delta E C} ; F g f 3^{-/}$
0.468018
0.538748
0.479024
0.435522
0.610028
0.608382
0.541674
0.584287
0.512067
0.468799
0.442253
0.43048
0.557548
0.520048
0.397543
0.481
0.500942
0.47514
0.606822

Unpaired t test $p<0.0001$
Middle panel

Vascular density (AU)

Ctrl

35.652

27.414

25.221

26.24

26.148

30.653

16.582

21.014

20.594

20.989

13.333

17.53

26.593

20.192

28.047

35.739

Fgfr1
10.546
16.313
$1 F g f r 3^{-/-}$
15.029
11.786
24.107
20.49

14.453

15.174

15.341

14.27

10.779

11.395

18.407

16.51

16.82

17.632

12.46

11.455

21.702
Right panel

Branch points $/ \mathrm{mm}^{2}$

$\begin{array}{cc}\text { Ctrl } & \text { Fgfr1 }{ }^{i \triangle E C} ; F^{\prime} g f r 3^{-/} \\ 644.875 & 211.375 \\ 478 & 289.875 \\ 546.875 & 272.375 \\ 480 & 178.5 \\ 641.875 & 289.375 \\ 634.25 & 430.625 \\ 392.875 & 282.375 \\ 359.875 & 350.5 \\ 462.25 & 242.75 \\ 415.625 & 221.5 \\ 299.75 & 189.25 \\ 258.5 & 167.75 \\ 376.625 & 259 \\ 367.75 & 284.625 \\ 465.3 & 160.5 \\ 515.6 & 181.5 \\ & 174.625 \\ & 184.625 \\ & 276.8\end{array}$

Unpaired t test

$p<0.0001$

Extended Data Figure 4. Requirement of endothelial Fgfr1/r3 for retinal angiogenesis.

a, Schematic of the experimental strategy to assess early formation of the retinal vasculature.

The red triangles indicate the intragastric injections of tamoxifen at P0, P1 and P2. b,

Representative images of isolectin B4 (IB4)-stained retinal vessels in P5 Fgfr ${ }^{i \Delta E C} ; \mathrm{Fgfr}^{-/-}$ and control mice. Scale bar, $500 \mu \mathrm{m}$. c, Quantification of vascular progression ( $\mathrm{d}$ is the distance between the vascular front and the optic nerve; $\mathrm{D}$ is the retina radius), vascular density (AU, arbitrary unit) and the number of branch points per $\mathrm{mm}^{2}$ retina area. $n=16$ retinas for control ( Fgfr $\left.1^{\text {flox/flox }} ; \mathrm{Fgfr}^{-/-}\right) ; n=19$ retinas for $F g f r 1^{i \Delta E C} ; \mathrm{Fgfr}^{-/}$. d, Angiogenic fronts of IB4- and Erg1/2/3-stained retinal vessels in P5 Fgfr ${ }^{i \Delta E C} ; \mathrm{Fgfr}^{-/-}$and control mice. Scale bar, $50 \mu \mathrm{m}$. e, Quantification of the number of tip cells per $200-\mu \mathrm{m}$ length ( $n=6$ retinas for control ( $\left.F g f r 1^{\text {flox/flox }} ; \mathrm{Fgfr}^{-/-}\right) ; n=4$ retinas for $F g f r 1^{i \Delta E C} ; \mathrm{Fgfr}^{-{ }^{-/}}$) and the number of vascular front endothelial cells (ECs) per $0.04-\mathrm{mm}^{2}$ retina area $(n=4$

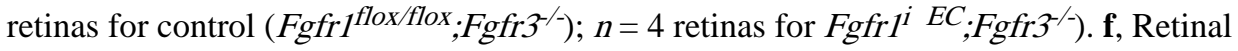
vessels stained for Phospho-Histone H3 (PH3) and IB4 in P5 Fgfr $1^{i \Delta E C} ; F g f r^{-/-}$and control 
mice. Scale bar, $50 \mu \mathrm{m}$. g, Quantification of the number of $\mathrm{PH}^{+} \mathrm{IB}^{+}$ECs per vascular area (normalized to control mice; $n=4$ retinas for control ( fgfr $\left.1^{\text {flox } / f l o x} ; \mathrm{Fgfr}^{-/}\right) ; n=6$ retinas

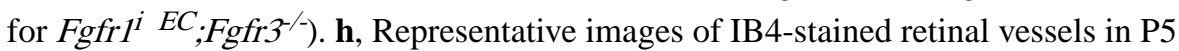
$F g f r 1^{i \Delta E C}$ and $F g f r 1^{\text {flox/flox }}$ mice. Scale bar, $500 \mu \mathrm{m}$. i, Quantification of vascular density and the number of branch points per $\mathrm{mm}^{2}$ retina area ( $n=18$ retinas for Fgfr $1^{\text {flox } / f l o x} ; n=8$ retinas for $F g f r 1^{i \Delta E C}$ ). j, Representative images of IB4-stained retinal vessels in P5 Fgfr ${ }^{\text {flox/flox }} ; \mathrm{Fgfr}^{+/-}$and $\mathrm{Fgfr} \mathrm{I}^{\text {flox/flox }} ; \mathrm{Fgfr} 3^{-/-}$mice. Scale bar, $500 \mu \mathrm{m}$. k, Quantification of vascular density and the number of branch points per $\mathrm{mm}^{2}$ retina area $(n=16$ retinas for Fgfr ${ }^{\text {flox/flox }} ; \mathrm{Fgfr}^{+/-} ; n=16$ retinas for $\mathrm{Fgfr} \mathrm{flox}^{\text {flofox }} ; \mathrm{Fgfr}^{-3^{--}}$). Data represent mean \pm s.e.m., ** $P<0.01, * * * P<0.001, \mathrm{~ns}=$ non-significant, calculated by unpaired $\mathrm{t}$ test $(\mathbf{c}, \mathbf{e}, \mathbf{g}, \mathbf{i}, \mathbf{k})$. 
a

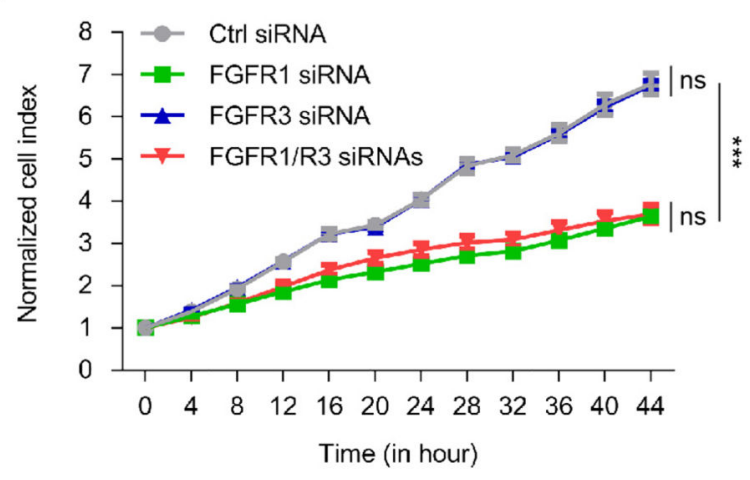

b

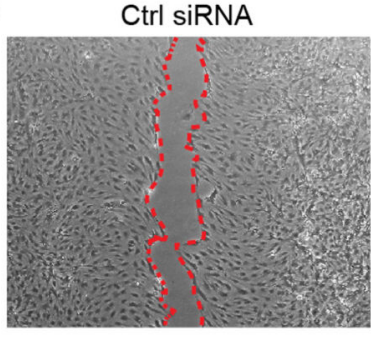

FGFR1 siRNA
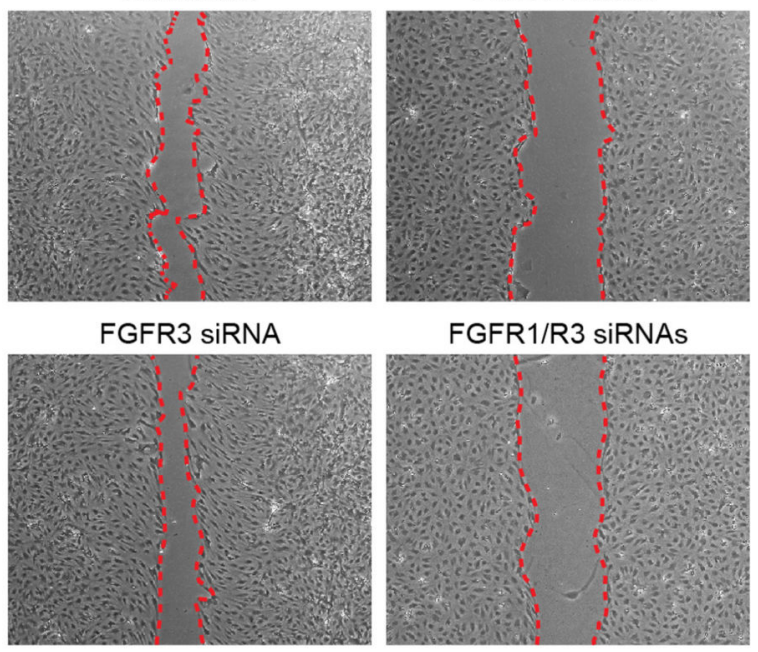

FGFR1/R3 siRNAs

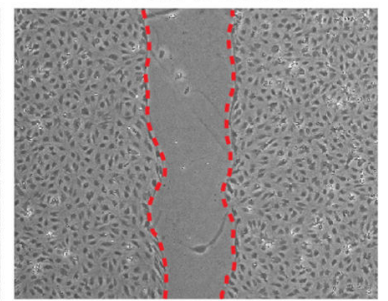

c
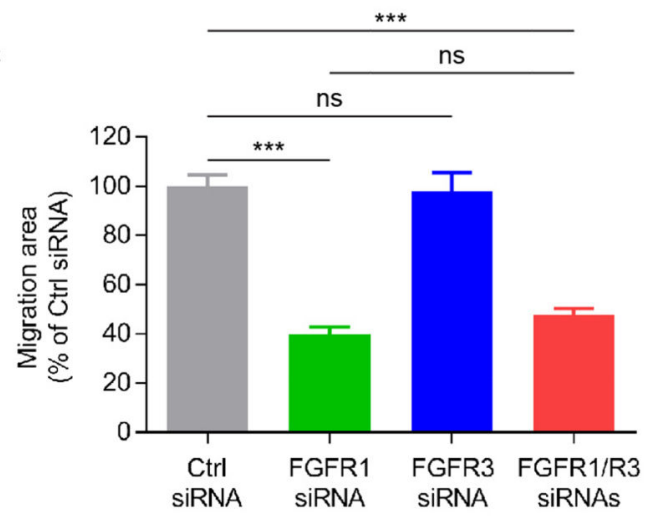

Extended Data Figure 5. Proliferation and migration of HDLECs with FGFR1 and/or FGFR3 knockdown.

a, Proliferation of HDLECs treated with indicated siRNAs was measured by using xCELLigence (see Methods) ( $n=4$ wells of samples for each condition; representative of 2 independent experiments). b, Wound healing assay to assess the migration of HDLECs transfected with siRNAs as indicated. Red dotted lines outline wound area in the last time point images of HDLECs with different treatments. c, Wound closure area between the first time point and the last time point was measured and normalized to that of control siRNA treated HDLECs $(n=8$ imaging fields for control siRNA, FGFR1 siRNA and FGFR3 
siRNA; $n=7$ imaging fields for FGFR1/R3 siRNAs; representative of 2 independent experiments). Data represent mean \pm s.e.m., $* * * P<0.001, \mathrm{~ns}=$ non-significant, calculated by One-way ANOVA plus Tukey's multiple comparisons test (a, c).

a

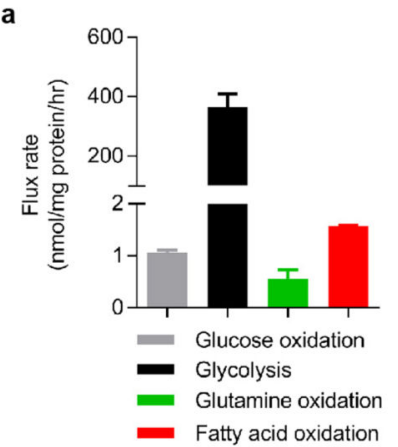

d

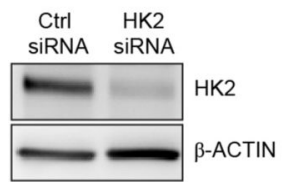

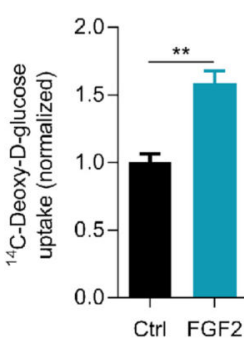

c

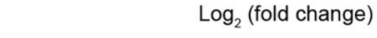

FGFR1 siRNA/Ctrl siRNA FGF2/Ctrl

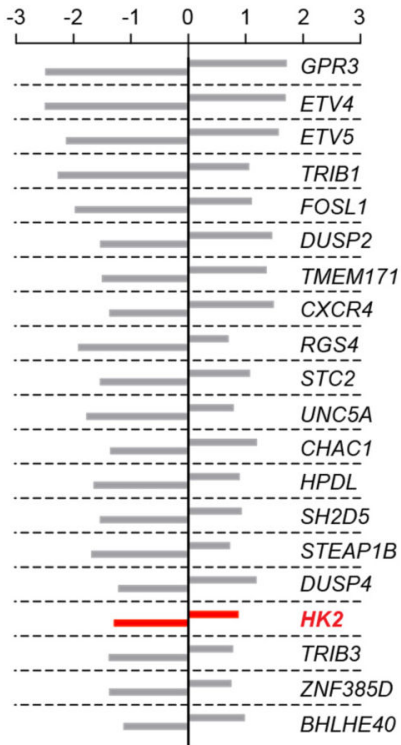

f

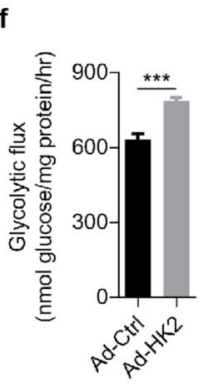

j

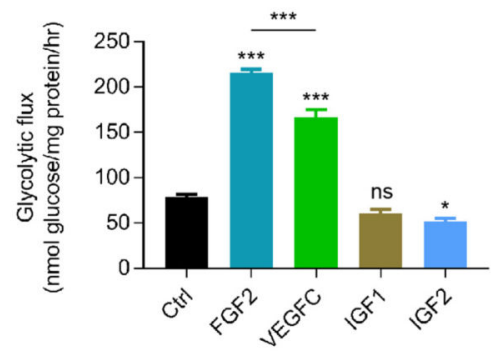

h

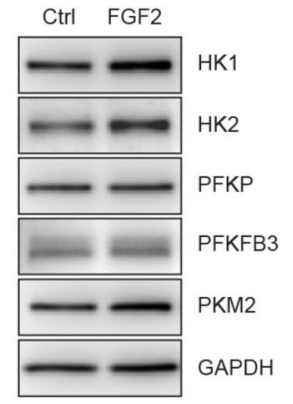

i

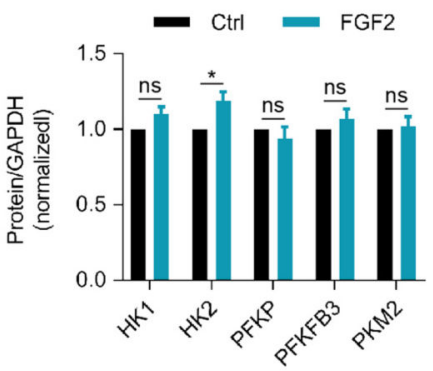

k

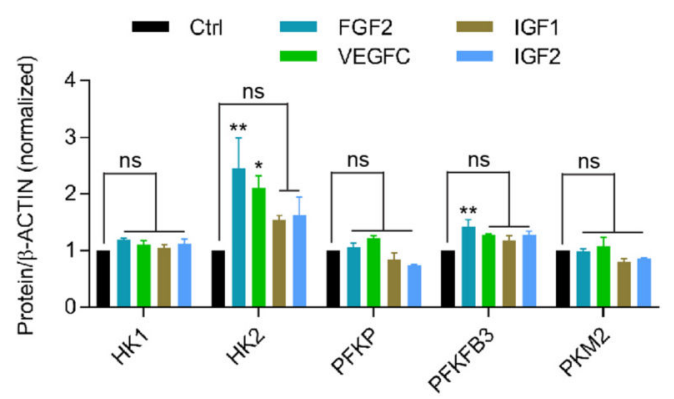

Extended Data Figure 6. Metabolic measurement and glycolytic enzyme expression in HDLECs and HUVECs.

a, Measurement of flux rate of different metabolic processes in HDLECs (glucose oxidation, $n=3$ samples; glycolysis, $n=11$ samples, combined from 3 experiments; glutamine oxidation, $n=5$ samples, combined from 2 experiments; and fatty acid oxidation, $n=4$ 
samples). b, Measurement of glucose uptake in HDLECs treated with or without FGF2 ( $n=$ 3 wells of samples for each condition). c, Top 20 protein-coding transcripts (ranked by fold change) which were increased by FGF2 and reduced by FGFR1 siRNA (see methods for details). $H K 2$, highlighted in red, is the only glucose metabolic gene in this list. d, Western blots showing the knockdown efficiency of HK2 siRNA. e, f, Measurement of glycolytic flux rate of HDLECs with indicated treatments. For $\mathbf{e}, n=4$ wells of samples for control siRNA, $n=4$ wells of samples for HK2 siRNA, $n=3$ wells of samples for control siRNA + FGF2, and $n=4$ wells of samples for HK2 siRNA + FGF2. For $\mathbf{f}, n=4$ wells of samples for each condition. g, Measurement of glycolytic flux rate of HUVECs in the absence or presence of FGF2 ( $n=4$ wells of samples for each condition, representative of 2 independent experiments). $\mathbf{h}, \mathbf{i}$, Representative western blot analysis (h) and densitometric quantification (i; $n=5$ replicates from 3 experiments) of glycolytic enzyme expression in control or FGF2-treated HUVECs. $\mathbf{j}$, Measurement of glycolytic flux rate of HDLECs treated with or without different growth factors ( $n=4$ wells of samples for each condition). $\mathbf{k}$, Densitometric quantification ( $n=3$ independent experiments) of glycolytic enzyme expression in HDLECs in the presence or absence of different growth factors. Data represent mean \pm s.e.m., $* P<0.05, * * P<0.01, * * * P<0.001, \mathrm{~ns}=$ non-significant, calculated by unpaired t test $(\mathbf{b}, \mathbf{f}, \mathbf{g}, \mathbf{i})$ and One-way ANOVA plus Sidak's $(\mathbf{e}, \mathbf{j})$ or Dunnett's $(\mathbf{k})$ multiple comparisons test. For gel source data, see Supplementary Fig. 1. 


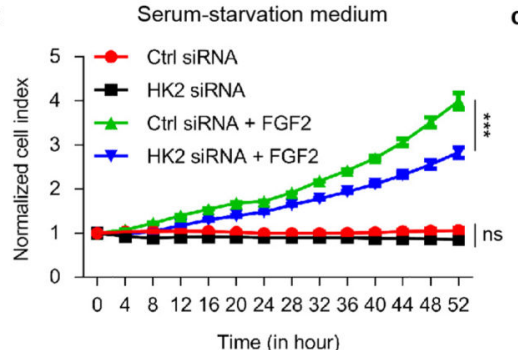

b
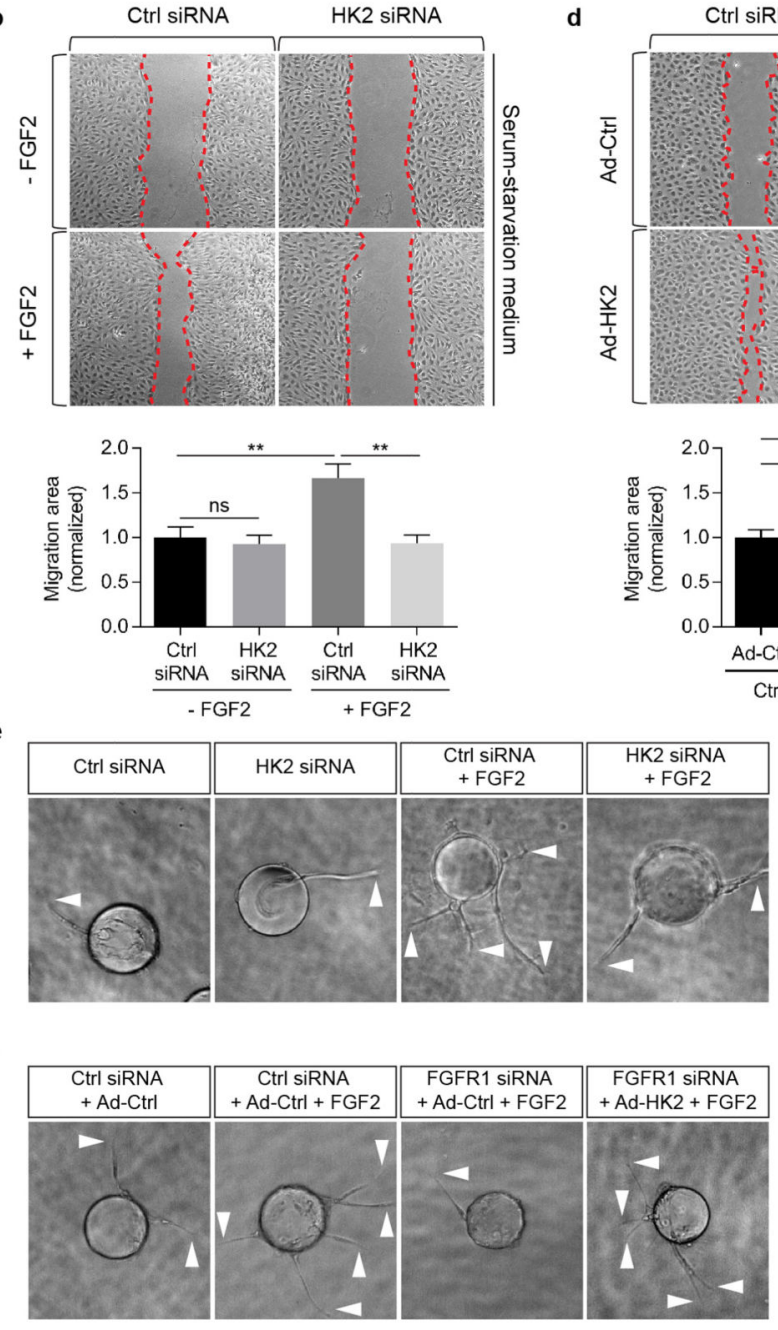

Fully supplemented medium

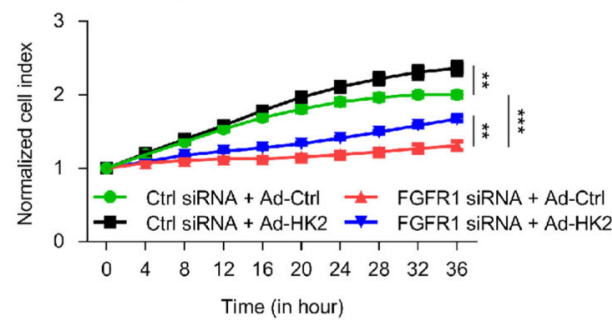

d
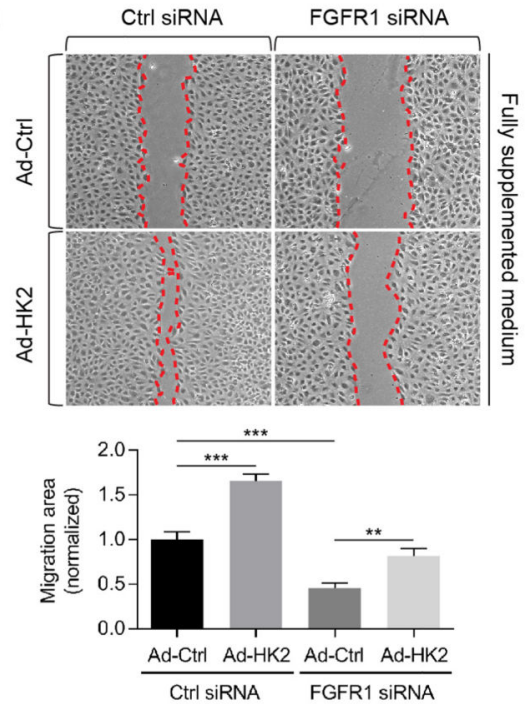
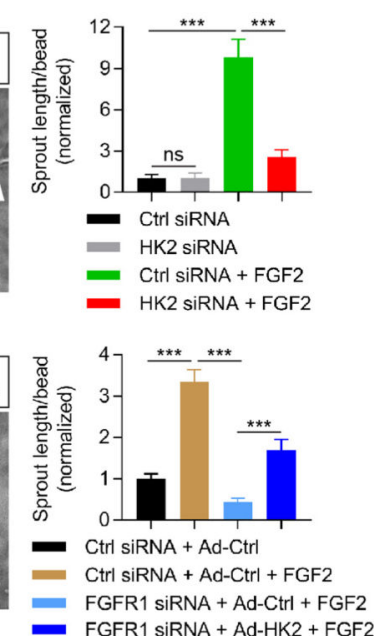

Extended Data Figure 7. Role of HK2 in FGF-dependent cellular behaviors.

$\mathbf{a}, \mathbf{b}$, Proliferation (a; $n=4$ wells of samples for each condition) and migration (b; $n=4$ imaging fields for each condition) of HDLECs with indicated siRNAs which were serumstarved and treated with or without FGF2. c, d, Proliferation (c; $n=4$ wells of samples for control siRNA + Ad-control, control siRNA + Ad-HK2 and FGFR1 siRNA + Ad-control; $n$ $=3$ wells of samples for FGFR1 siRNA + Ad-HK2) and migration $(\mathbf{d} ; n=8$ imaging fields for each condition) of HDLECs with indicated siRNAs which were cultured in fully supplemented medium and treated with control or HK2 adenovirus. Proliferation was 
measured using xCELLigence and migration was analyzed through wound healing assay (see Methods). Wound closure area between the first time point and the last time point was measured and normalized to that of control siRNA treated HDLECs (b) or HDLECs treated with control siRNA and control adenovirus (d). Red dotted lines outline wound area in the last time point images of HDLECs with different treatments. e, f, Representative images and quantification of microcarrier beads coated with HDLECs under treatments as indicated. Total length of LEC sprouts per bead was quantified. For e, $n=14$ beads for control siRNA, $n=14$ beads for HK2 siRNA, $n=19$ beads for control siRNA + FGF2, and $n=25$ beads for HK2 siRNA + FGF2. For $\mathbf{f}, n=25$ beads for each condition. Data represent mean \pm s.e.m., $* * P<0.01, * * * P<0.001, \mathrm{~ns}=$ non-significant, calculated by One-way ANOVA plus Sidak's multiple comparisons test (a-f). 
a
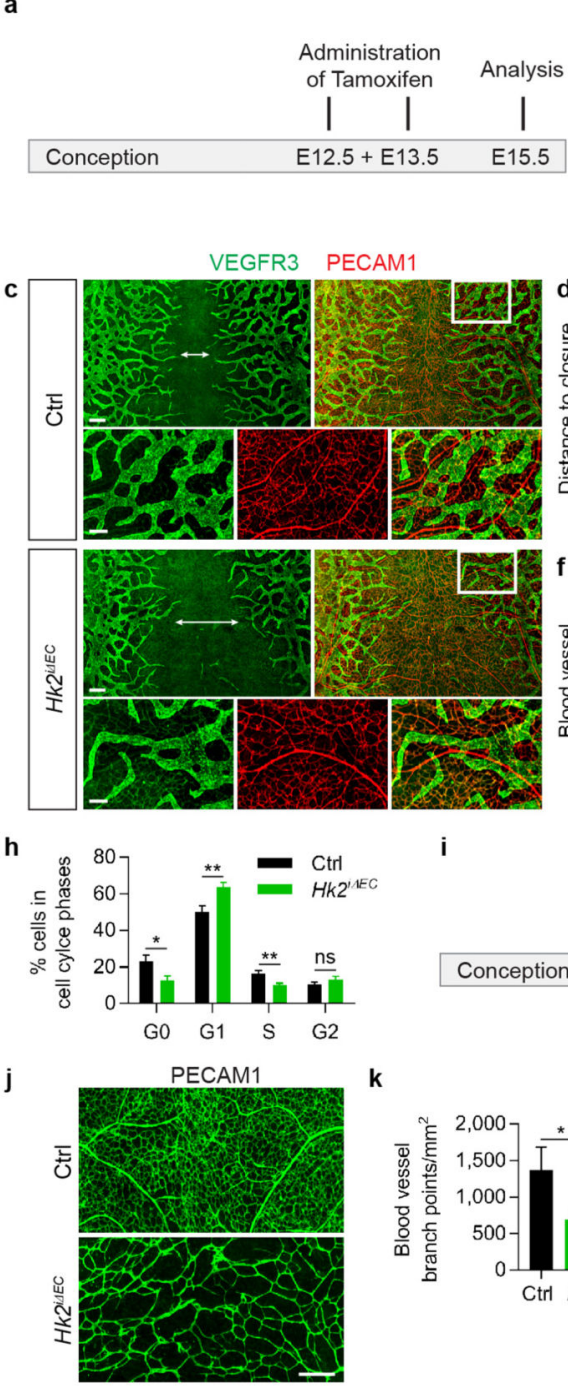

i
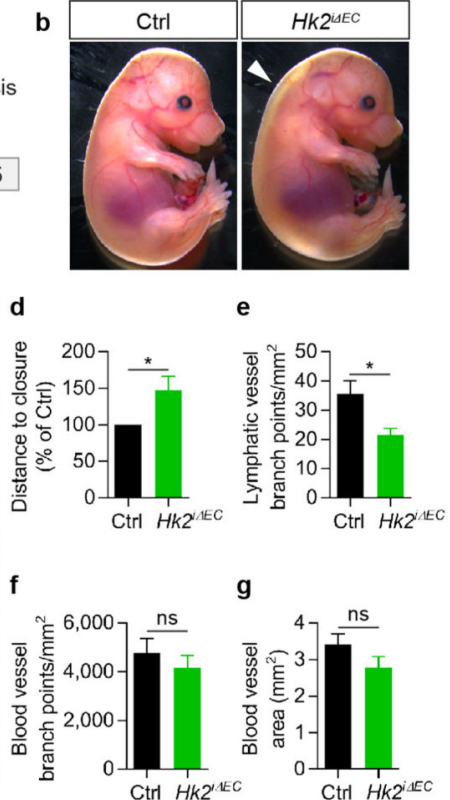

g
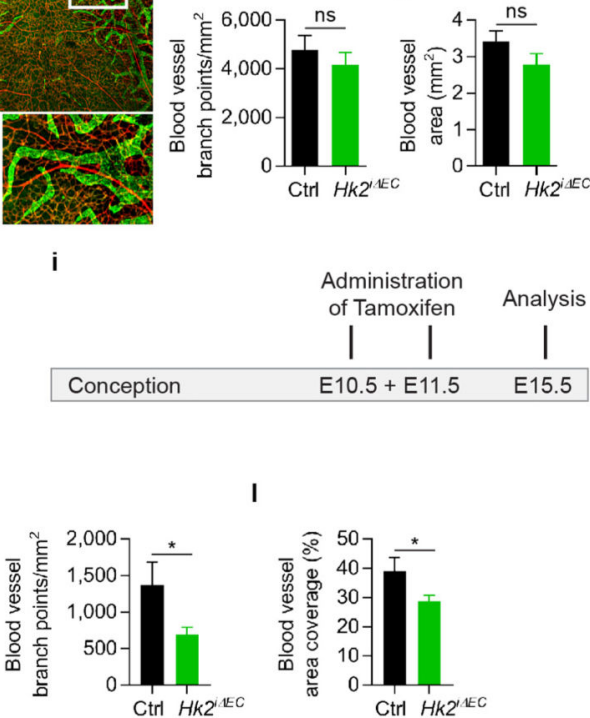

m
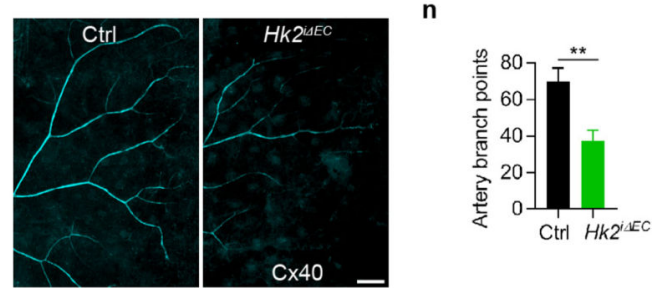

Extended Data Figure 8. Effect of endothelium-specific deletion of $\mathrm{Hk} 2$ on the lymphatic and blood vessel development in the skin.

a, Schematic of the experimental strategy. b, Bright-field images of E15.5 Hk2 $2^{i \Delta E C}$ and control embryos treated with tamoxifen at E12.5 and E13.5. Arrowhead denotes area with lymphedema. c, Representative images of anterior dorsal skin with VEGFR3 and PECAM1 staining from E15.5 Hk2 $2^{i \Delta E C}$ and control ( $\left.H k 2^{\text {flox/flox }}\right)$ embryos with tamoxifen treatment at E12.5 and E13.5. Double-headed arrows indicate the distance between the two leading fronts of the lymphatic vessels, which is larger in $H k 2^{i \Delta E C}$ skin than in control. Lower 
panels (scale bar, $100 \mu \mathrm{m}$ ) for each genotype are high-magnification images of boxed regions in top panels (scale bar, $250 \mu \mathrm{m}$ ). d, e, Quantification of the distance between the two leading fronts of the lymphatic vessels (d; $n=4$ litters) and the number of lymphatic branch points per $\mathrm{mm}^{2}$ skin area $\left(\mathbf{e} ; n=5\right.$ embryos for control $\left(H k 2^{\text {flox } f l o x}\right) ; n=6$ embryos for $H k 2^{i \Delta E C}$ ). $\mathbf{f}, \mathbf{g}$, Quantification of the number of blood vessel branch points per $\mathrm{mm}^{2}$ skin area (f) and area covered by blood vessels (g). $n=5$ embryos for control $\left(H k 2^{\text {flox/flox }}\right) ; n=6$ embryos for $H k 2^{i \Delta E C}$. h, LECs were isolated from E15.5 control $\left(H k 2^{\text {flox/flox }}\right)$ and $H k 2^{i \Delta E C}$ embryos and analyzed for cell cycle distribution. Percentage of cells in different cell cycle

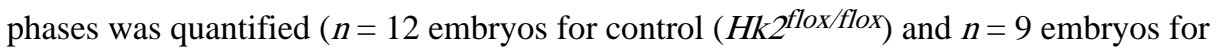
$H k 2^{i \Delta E C}$ ). i, The experimental strategy. $\mathbf{j}$, Representative images of anterior dorsal skin with PECAM1 staining from E15.5 Hk $2^{i \Delta E C}$ and control embryos with tamoxifen treatment at E10.5 and E11.5. Scale bar, $250 \mu \mathrm{m} . \mathbf{k}, \mathbf{l}$, Quantification of the number of blood vessel branch points per $\mathrm{mm}^{2}$ skin area $(\mathbf{k})$ and blood vessel covered area relative to skin area (I). $n$ $=4$ embryos for control $\left(H k 2^{\text {flox/flox }}\right) ; n=7$ embryos for $H k 2^{i \Delta E C}$. $\mathbf{m}$, Anterior dorsal skin stained for $\mathrm{Cx} 40$ in E15.5 Hk2 ${ }^{i \Delta E C}$ and control embryos treated with tamoxifen at E10.5 and E11.5. Scale bar, $250 \mu \mathrm{m}$. $\mathbf{n}$, Quantification of the number of artery branch points ( $n=4$ embryos for control $\left(H k 2^{f l o x} / f l o x\right) ; n=7$ embryos for $\left.H k 2^{i \Delta E C}\right)$. Data represent mean \pm s.e.m., $* P<0.05, * * P<0.01, \mathrm{~ns}=$ non-significant, calculated by unpaired t test $(\mathbf{d}, \mathbf{e}, \mathbf{f}-\mathbf{h}$, $\mathbf{k}, \mathbf{l}, \mathbf{n})$. 
a

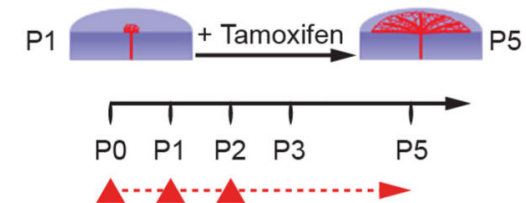

C

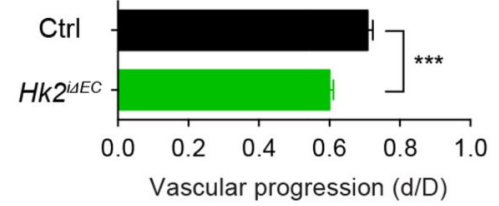

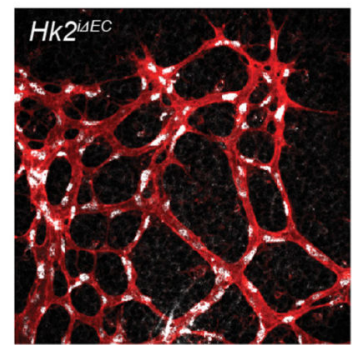

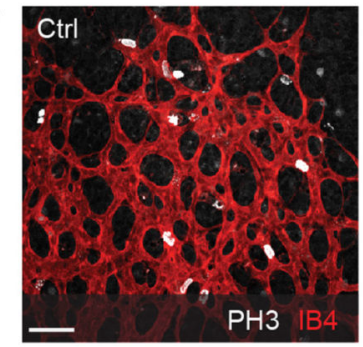

h

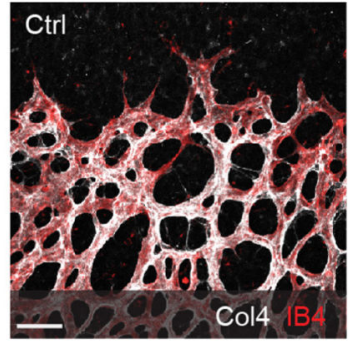

b

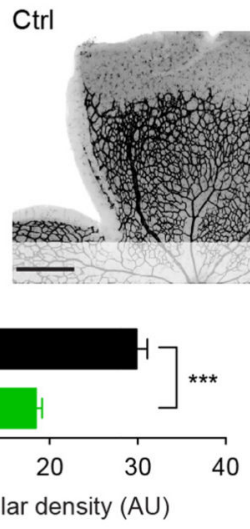

$H k 2^{i \Delta E C}$

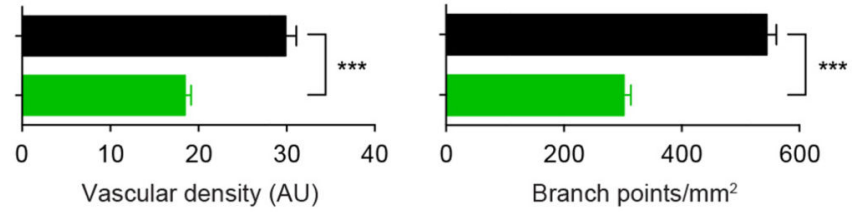

e Ctrl
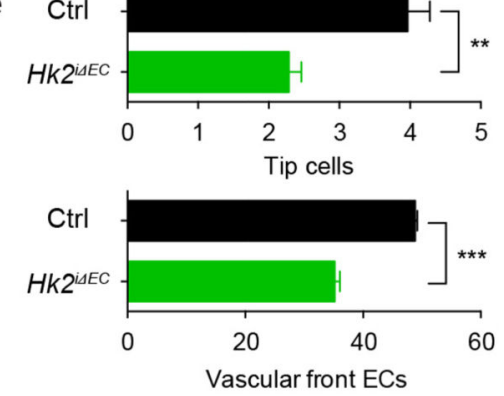

g

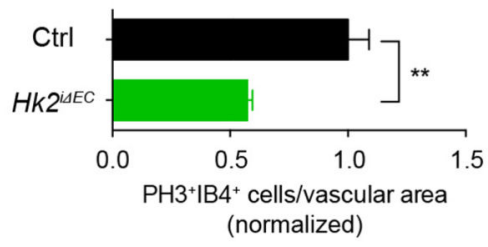

i

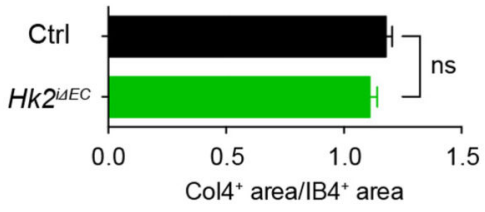

Extended Data Figure 9. Endothelial Hk2 is required for retinal angiogenesis.

a, Schematic of the experimental strategy to assess early formation of the retinal vasculature (P0-P5). The red triangles indicate the intragastric injections of tamoxifen at P0, P1 and P2. b, Representative images of IB4-stained retinal vessels in P5 $H k 2^{i \Delta E C}$ and control mice. Scale bar, $500 \mu \mathrm{m}$. c, Quantification of vascular progression ( $\mathrm{d}$ is the distance between the vascular front and the optic nerve; $\mathrm{D}$ is the retina radius), vascular density (AU, arbitrary unit) and the number of branch points per $\mathrm{mm}^{2}$ retina area. $n=18$ retinas for control $\left(H k 2^{f l o x} / f l o x\right)$ and $n=24$ retinas for $H k 2^{i \Delta E C}$. d, Angiogenic fronts of IB4- and Erg1/2/3- 
stained retinal vessels in P5 $H k 2^{i \Delta E C}$ and control mice. Scale bar, $50 \mu \mathrm{m}$. e, Quantification of the number of tip cells per 200- $\mu$ m length ( $n=4$ retinas for control $\left(H k 2^{\text {flox } / f l o x}\right) ; n=4$ retinas for $H k 2^{i \Delta E C}$ ) and the number of vascular front ECs per $0.04-\mathrm{mm}^{2}$ retina area $(n=4$ retinas for control $\left(H k 2^{\text {flox/flox }}\right) ; n=4$ retinas for $H k 2^{i \Delta E C}$ ). f, Retinal vessels stained for PH3 and IB4 in P5 Hk2 $2^{i \Delta E C}$ and control mice. Scale bar, $50 \mu \mathrm{m}$. g, Quantification of the number of $\mathrm{PH} 3^{+} \mathrm{IB} 4^{+}$ECs per vascular area (normalized to control mice; $n=4$ retinas for control $\left(H k 2^{\text {flox } / f l o x}\right) ; n=4$ retinas for $\left.H k 2^{i \Delta E C}\right)$. h, Staining for Collagen IV (Col4) and IB4 in the retinas of P5 $H k 2^{i \Delta E C}$ and control mice. Scale bar, $50 \mu \mathrm{m}$. i, Quantification of Col $4^{+}$ area per IB $4^{+}$area $\left(n=6\right.$ retinas for control $\left(H k 2^{\text {flox } / f l o x}\right) ; n=8$ retinas for $\left.H k 2^{i \Delta E C}\right)$. Data represent mean \pm s.e.m., $* * P<0.01$, $* * * P<0.001, \mathrm{~ns}=$ non-significant, calculated by unpaired t test $(\mathbf{c}, \mathbf{e}, \mathbf{g}, \mathbf{i})$. 
a
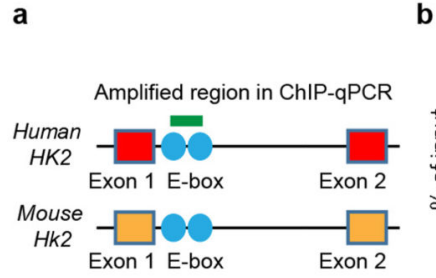
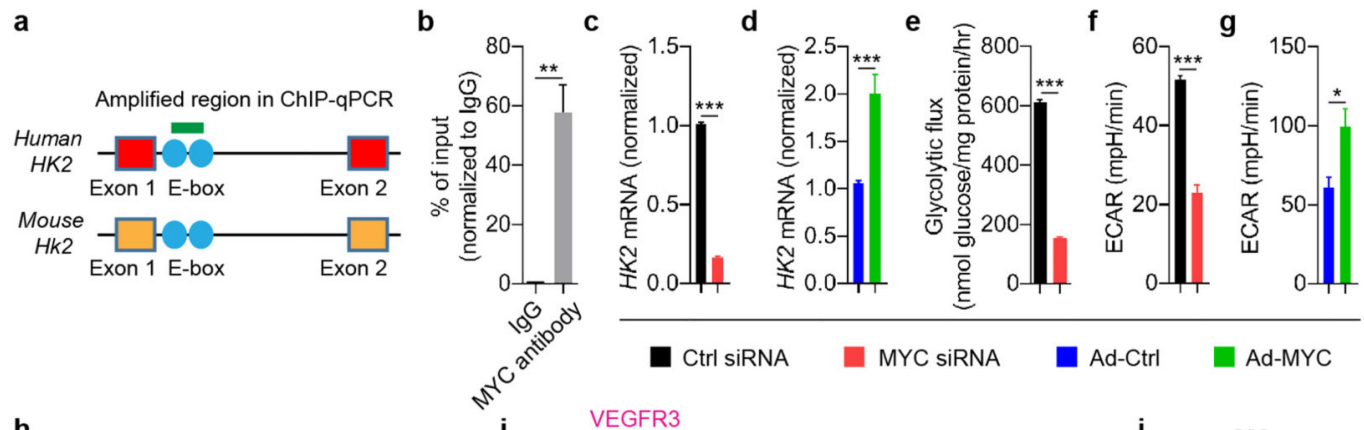

Ctrl siRNA

MYC siRNA

Ad-Ctrl

Ad-MYC

h
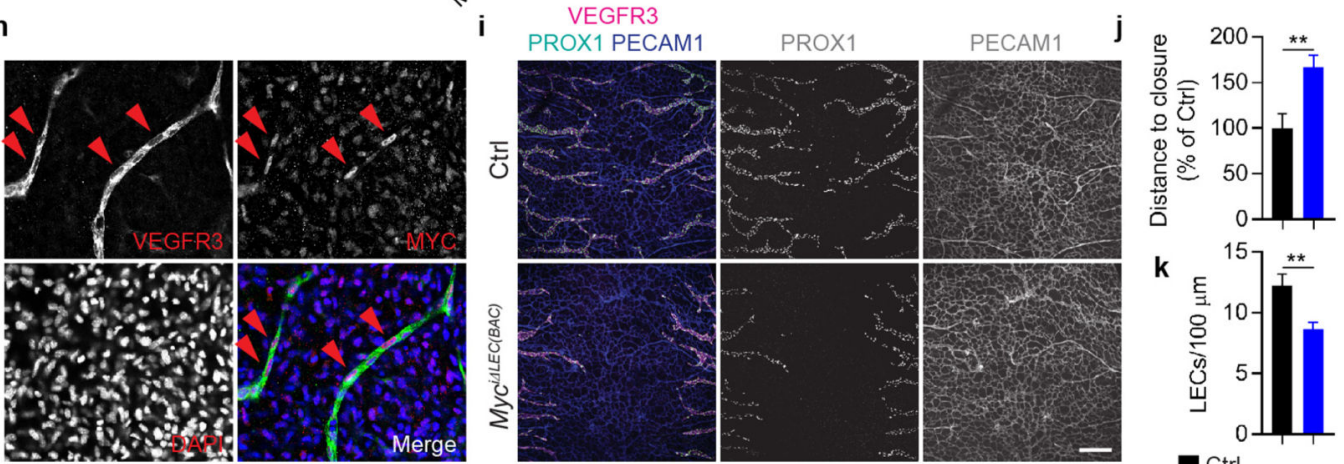

I

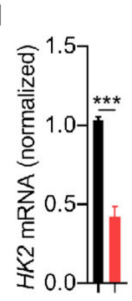

m

n
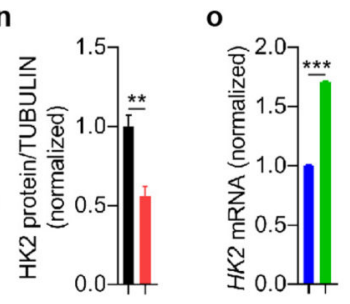

p

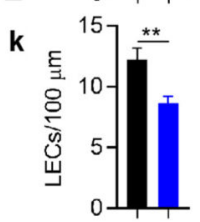

$\square \mathrm{Ctrl}$

MYC $C^{\text {SLEC(BAC) }}$

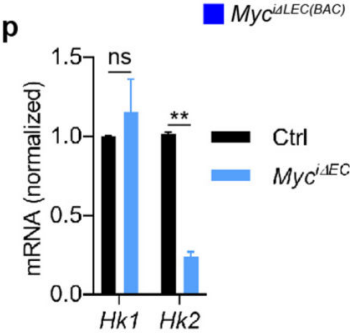

Ctrl siRNA $\quad$ MYC siRNA $\square$ Ad-Ctrl $\square$ Ad-MYC

q
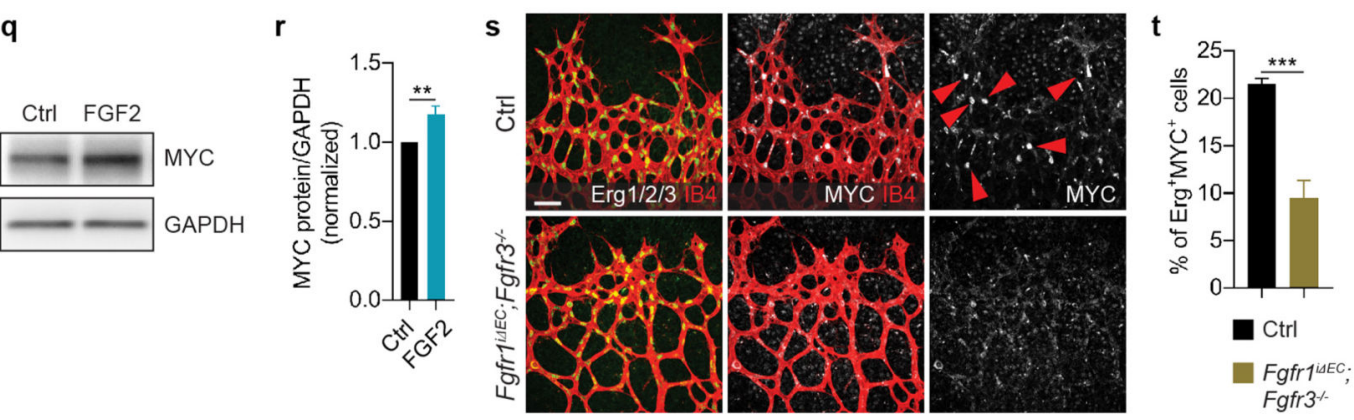

Extended Data Figure 10. Characterization of FGF-MYC-HK2 signaling in endothelial cells. a, Schematic showing that E-boxes, which are MYC binding elements, localize in the first intron of human and mouse $H K 2$ genes. Primers were designed to amplify the E-box containing region (green bar) in ChIP-qPCR assay. b, ChIP-qPCR analysis of DNA immunoprecipitated with MYC antibody or $\operatorname{IgG}$ ( $n=3$ independent experiments) in HDLECs. c, qPCR analysis of $H K 2$ expression in HDLECs transfected with control siRNA or MYC siRNA ( $n=4$ experiments). d, qPCR analysis of $H K 2$ mRNA in HDLECs infected with control or MYC adenovirus ( $n=6$ experiments). e, Glycolytic flux measurement of 
HDLECs transfected with control siRNA or MYC siRNA ( $n=4$ wells of samples for each treatment, representative of 2 independent experiments). $\mathbf{f}$, Extracellular acidification rate (ECAR) in HDLECs transfected with control siRNA or MYC siRNA. $n=6$ replicates (3 independent experiments, duplicates per experiment). g, ECAR in HDLECs infected with control or MYC adenovirus. $n=4$ replicates ( 2 independent experiments, duplicates per experiment). h, Anterior dorsal skin was dissected from E15.5 mouse embryos and immunostained with anti-VEGFR3 and anti-MYC antibodies. i, Confocal images of anterior dorsal skin with VEGFR3, PROX1 and PECAM1 staining from E15.5 Myc ${ }^{i \Delta L C C(B A C)}$ and control embryos. Scale bar, $250 \mu \mathrm{m}$. j, k, Quantification of the distance between the leading fronts of ingrowing lymphatics ( $\mathbf{j} ; n=7$ embryos for control (Myclox/flox $) ; n=9$ embryos for $M y c^{i \triangle L E C(B A C)}$ ) and the number of LECs (PROX1 staining) per 100- $\mu \mathrm{m}$ length of lymphatic vessels $\left(\mathbf{k} ; n=6\right.$ embryos for control $\left(M y c^{\text {flox } / f l o x}\right) ; n=8$ embryos for $\left.M y c^{i \Delta L E C(B A C)}\right) . \mathbf{l}, \mathrm{qPCR}$ analysis of $H K 2$ expression in HUVECs transfected with control siRNA or MYC siRNA ( $n=4$ experiments). m, n, Representative immunoblot analysis (m) and densitometric quantification (n) of HK2 expression in HUVECs transfected with control siRNA or MYC siRNA ( $n=4$ independent experiments). o, qPCR analysis of $H K 2$ mRNA in HUVECs infected with control or MYC adenovirus ( $n=2-4$ experiments). p, qPCR analysis of $H k 1$ and $H k 2$ expression in dermal BECs isolated from E15.5 Myc ${ }^{i \Delta E C}$ and control embryos with tamoxifen treatment at E11.5 and E12.5 ( $n=2-4$ embryos). q, r, Representative western blot (q) and densitometric quantification (r) of MYC expression in HUVECs treated with or without FGF2 ( $n=5$ replicates from 3 experiments). $\mathbf{s}$,

Representative images showing Myc expression was reduced in retinal vasculature of P5 Fgfr $1^{i \Delta E C} ; F g f r^{-/-}$compared with control (tamoxifen treatment from P0 to P2). Scale bar, 50 $\mu \mathrm{m}$. $\mathbf{t}$, Quantification of the percentage of $\mathrm{MYC}^{+}$retinal ECs in the vascular fronts $(n=4$ retinas for each genotype). Data represent mean \pm s.e.m., $* P<0.05$, ** $P<0.01, * * * P<$ 0.001, ns = non-significant, calculated by unpaired t test $(\mathbf{b}-\mathbf{g}, \mathbf{j}-\mathbf{l}, \mathbf{n}-\mathbf{p}, \mathbf{r}, \mathbf{t})$. For gel source data, see Supplementary Fig. 1.

\section{Supplementary Material}

Refer to Web version on PubMed Central for supplementary material.

\section{Acknowledgement}

We thank Ralf Adams for Cdh5-CreER T2, Taija Makinen for Prox1-CreER T2(BAC), Guillermo Oliver for Prox1$\mathrm{CreER}{ }^{T 2(K I)}$, as well as Flora Vaccarino and Simone Tomasi for $\mathrm{Fgfr} \mathrm{flox}^{\mathrm{flox}} ; \mathrm{Fgfr}^{+{ }^{+/}}{ }^{-}$mice. We also would like to thank Hossein Ardehali for providing HK2 adenovirus, Brian G. Coon for generating CRISPR/Cas9 lentivirus, Koen van den Dries for real-time imaging and analysis of cell motility, Guy Eelen and Annelies Quaegebeur for metabolic analysis, Timur Yarovinsky for instruction and assistance on the flow cytometry, Andrew Kuo for help with the Seahorse metabolic analyzer, and Fu Zhuo for schematic illustrations. PY is funded by American Heart Association Postdoctoral Fellowship (15POST25830021). JZ was funded by the Brown-Coxe Fellowship from Yale University. FDS was supported as a postdoctoral researcher by the Research Foundation Flanders (FWO). PC is funded by IUAP P7/03, long-term structural Methusalem funding by the Flemish Government, FWO G.0598.12, G. 0532.10, G.0817.11, G.0834.13, 1.5.202.10.N Krediet aan navorsers, Leducq Transatlantic Network Artemis, AXA Research Fund (1465) and Foundation against Cancer, ERC Advanced Research Grant (EU-ERC269073, PC). MP is supported by the Max Planck Society, the European Research Council (ERC) Starting Grant ANGIOMET (311546), the Deutsche Forschungsgemeinschaft (SFB 834), the Excellence Cluster Cardiopulmonary System (EXC 147/1), the LOEWE grant Ub-Net, the DZHK (German Center for Cardiovascular Research), and the European Molecular Biology Organization Young Investigator Programme. MS is supported by NIH grants HL053793, HL084619 and ARTEMIS Leducq Transatlantic Network. 


\section{References}

1. Eelen G, de Zeeuw P, Simons M, Carmeliet P. Endothelial Cell Metabolism in Normal and Diseased Vasculature. Circulation research. 2015; 116:1231-1244. DOI: 10.1161/CIRCRESAHA.116.302855 [PubMed: 25814684]

2. Simons M, Gordon E, Claesson-Welsh L. Mechanisms and regulation of endothelial VEGF receptor signalling. Nat Rev Mol Cell Biol. 2016; 17:611-625. DOI: 10.1038/nrm.2016.87 [PubMed: 27461391]

3. De Bock K, Georgiadou M, Carmeliet P. Role of endothelial cell metabolism in vessel sprouting. Cell metabolism. 2013; 18:634-647. DOI: 10.1016/j.cmet.2013.08.001 [PubMed: 23973331]

4. Ornitz DM, Itoh N. The Fibroblast Growth Factor signaling pathway. Wiley interdisciplinary reviews Developmental biology. 2015; 4:215-266. DOI: 10.1002/wdev.176 [PubMed: 25772309]

5. Wang Y, et al. Ephrin-B2 controls VEGF-induced angiogenesis and lymphangiogenesis. Nature. 2010; 465:483-486. [PubMed: 20445537]

6. Bazigou E, et al. Genes regulating lymphangiogenesis control venous valve formation and maintenance in mice. The Journal of clinical investigation. 2011; 121:2984-2992. DOI: 10.1172/ JCI58050 [PubMed: 21765212]

7. James JM, Nalbandian A, Mukouyama YS. TGFbeta signaling is required for sprouting lymphangiogenesis during lymphatic network development in the skin. Development. 2013; 140:3903-3914. DOI: 10.1242/dev.095026 [PubMed: 23946447]

8. Herbert C, et al. Molecular mechanism of SSR128129E, an extracellularly acting, small-molecule, allosteric inhibitor of FGF receptor signaling. Cancer cell. 2013; 23:489-501. DOI: 10.1016/j.ccr. 2013.02.018 [PubMed: 23597563]

9. De Bock K, et al. Role of PFKFB3-driven glycolysis in vessel sprouting. Cell. 2013; 154:651-663. DOI: 10.1016/j.cell.2013.06.037 [PubMed: 23911327]

10. Zheng W, Aspelund A, Alitalo K. Lymphangiogenic factors, mechanisms, and applications. The Journal of clinical investigation. 2014; 124:878-887. DOI: 10.1172/JCI71603 [PubMed: 24590272]

11. Patra KC, et al. Hexokinase 2 is required for tumor initiation and maintenance and its systemic deletion is therapeutic in mouse models of cancer. Cancer cell. 2013; 24:213-228. DOI: 10.1016/ j.ccr.2013.06.014 [PubMed: 23911236]

12. Srinivasan RS, et al. Lineage tracing demonstrates the venous origin of the mammalian lymphatic vasculature. Genes Dev. 2007; 21:2422-2432. DOI: 10.1101/gad.1588407 [PubMed: 17908929]

13. Cao R, et al. Mouse corneal lymphangiogenesis model. Nature protocols. 2011; 6:817-826. DOI: 10.1038/nprot.2011.359 [PubMed: 21637201]

14. Kim JW, Gao P, Liu YC, Semenza GL, Dang CV. Hypoxia-inducible factor 1 and dysregulated cMyc cooperatively induce vascular endothelial growth factor and metabolic switches hexokinase 2 and pyruvate dehydrogenase kinase 1. Mol Cell Biol. 2007; 27:7381-7393. DOI: 10.1128/MCB. 00440-07 [PubMed: 17785433]

15. Wilhelm K, et al. FOXO1 couples metabolic activity and growth state in the vascular endothelium. Nature. 2016; 529:216-220. DOI: 10.1038/nature16498 [PubMed: 26735015]

16. Ichise T, Yoshida N, Ichise H. FGF2-induced Ras/Erk MAPK signalling maintains lymphatic endothelial cell identity by up-regulating endothelial cell-specific gene expression and suppressing TGFbeta signalling via Smad2. Journal of cell science. 2013; doi: 10.1242/jcs.137836

17. Chen PY, et al. FGF regulates TGF-beta signaling and endothelial-to-mesenchymal transition via control of let-7 miRNA expression. Cell reports. 2012; 2:1684-1696. DOI: 10.1016/j.celrep. 2012.10.021 [PubMed: 23200853]

18. Oladipupo SS, et al. Endothelial cell FGF signaling is required for injury response but not for vascular homeostasis. Proc Natl Acad Sci U S A. 2014; 111:13379-13384. DOI: 10.1073/pnas. 1324235111 [PubMed: 25139991]

19. Murakami M, et al. The FGF system has a key role in regulating vascular integrity. The Journal of clinical investigation. 2008; 118:3355-3366. DOI: 10.1172/JCI35298 [PubMed: 18776942] 
20. Rash BG, Lim HD, Breunig JJ, Vaccarino FM. FGF signaling expands embryonic cortical surface area by regulating Notch-dependent neurogenesis. J Neurosci. 2011; 31:15604-15617. DOI: 10.1523/JNEUROSCI.4439-11.2011 [PubMed: 22031906]

21. de Alboran IM, et al. Analysis of C-MYC function in normal cells via conditional gene-targeted mutation. Immunity. 2001; 14:45-55. [PubMed: 11163229]

22. Muzumdar MD, Tasic B, Miyamichi K, Li L, Luo L. A global double-fluorescent Cre reporter mouse. Genesis. 2007; 45:593-605. DOI: 10.1002/dvg.20335 [PubMed: 17868096]

23. Dobin A, et al. STAR: ultrafast universal RNA-seq aligner. Bioinformatics. 2013; 29:15-21. DOI: 10.1093/bioinformatics/bts635 [PubMed: 23104886]

24. Li B, Dewey CN. RSEM: accurate transcript quantification from RNA-Seq data with or without a reference genome. BMC Bioinformatics. 2011; 12:323.doi: 10.1186/1471-2105-12-323 [PubMed: 21816040]

25. Robinson MD, McCarthy DJ, Smyth GK. edgeR: a Bioconductor package for differential expression analysis of digital gene expression data. Bioinformatics. 2010; 26:139-140. DOI: 10.1093/bioinformatics/btp616 [PubMed: 19910308]

26. Young MD, Wakefield MJ, Smyth GK, Oshlack A. Gene ontology analysis for RNA-seq: accounting for selection bias. Genome biology. 2010; 11:R14.doi: 10.1186/gb-2010-11-2-r14 [PubMed: 20132535]

27. Chittenden TW, et al. Therapeutic implications of GIPC1 silencing in cancer. PloS one. 2010; 5:e15581.doi: 10.1371/journal.pone.0015581 [PubMed: 21209904]

28. Chittenden TW, et al. nEASE: a method for gene ontology subclassification of high-throughput gene expression data. Bioinformatics. 2012; 28:726-728. DOI: 10.1093/bioinformatics/bts011 [PubMed: 22247278]

29. Alves TC, et al. Integrated, Step-Wise, Mass-Isotopomeric Flux Analysis of the TCA Cycle. Cell metabolism. 2015; 22:936-947. DOI: 10.1016/j.cmet.2015.08.021 [PubMed: 26411341]

30. Kibbey RG, et al. Mitochondrial GTP regulates glucose-stimulated insulin secretion. Cell metabolism. 2007; 5:253-264. DOI: 10.1016/j.cmet.2007.02.008 [PubMed: 17403370]

31 . Wu R, et al. Reduction in hexokinase II levels results in decreased cardiac function and altered remodeling after ischemia/reperfusion injury. Circulation research. 2011; 108:60-69. DOI: 10.1161/CIRCRESAHA.110.223115 [PubMed: 21071708]

32. Dubrac A, et al. Targeting NCK-Mediated Endothelial Cell Front-Rear Polarity Inhibits Neovascularization. Circulation. 2016; 133:409-421. DOI: 10.1161/CIRCULATIONAHA. 115.017537 [PubMed: 26659946]

33. Tang Z, et al. A mouse model of the cornea pocket assay for angiogenesis study. Journal of visualized experiments : JoVE. 2011; doi: 10.3791/3077 
a

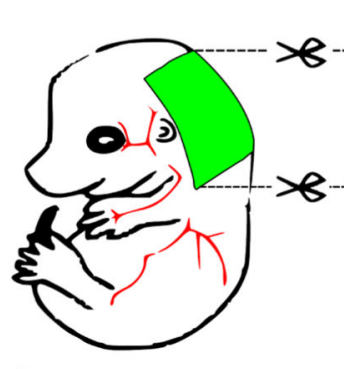

b

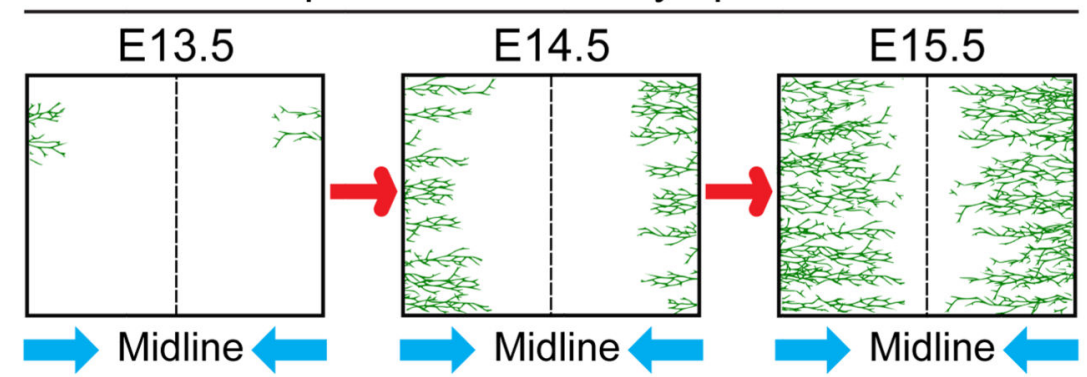

C
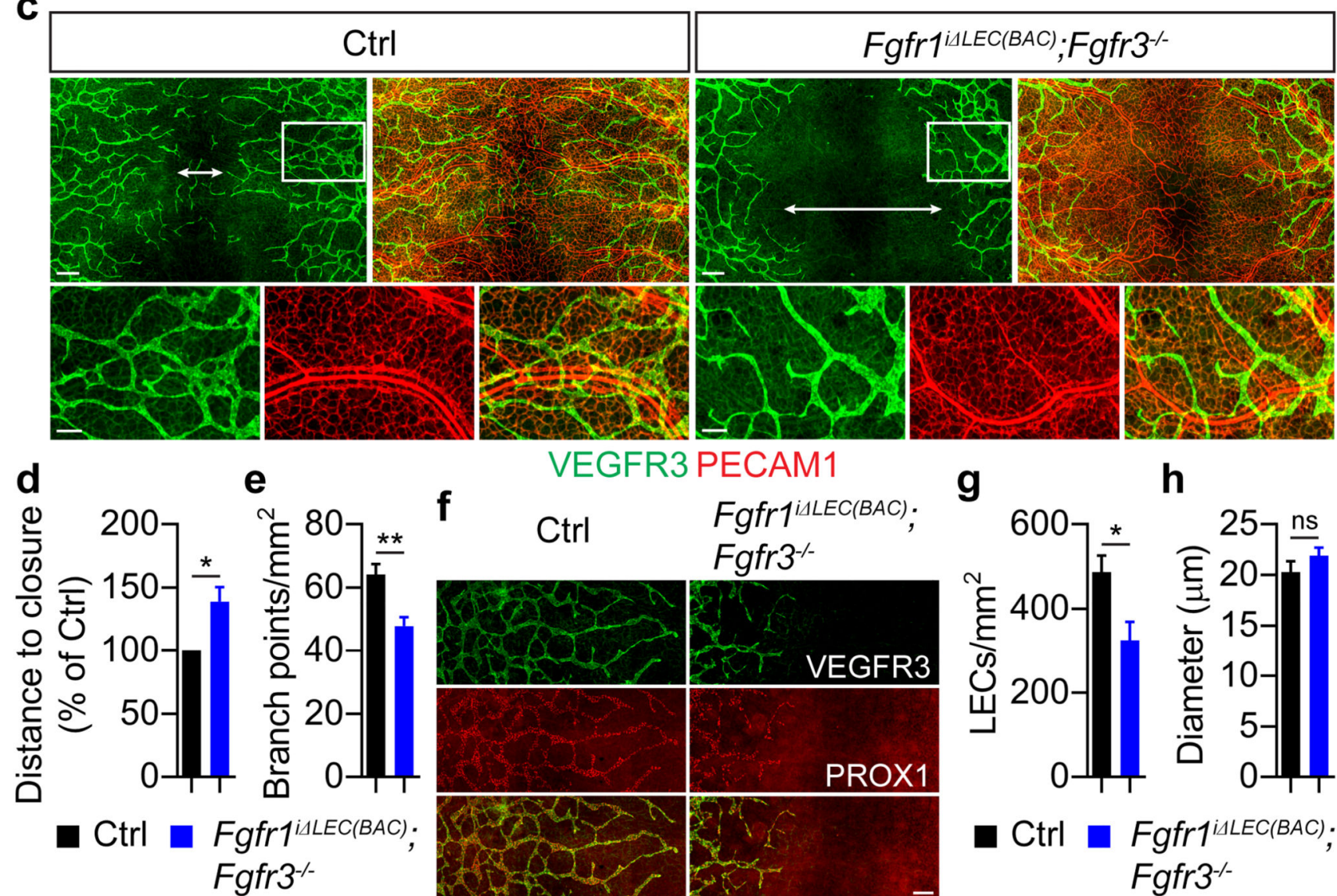

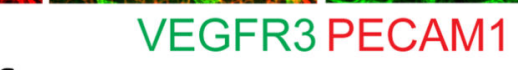

C Ctrl

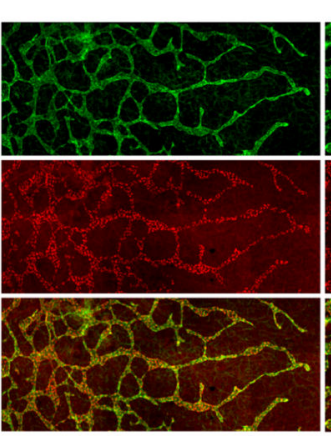

Fgfr1 ${ }^{\mathrm{ILLEC}(B A C)}$; Fgfr $^{-1-}$

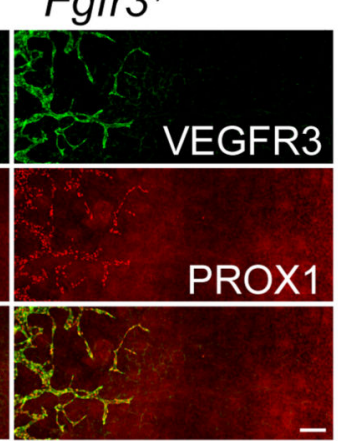

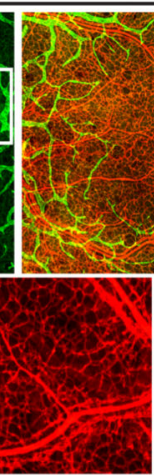

g

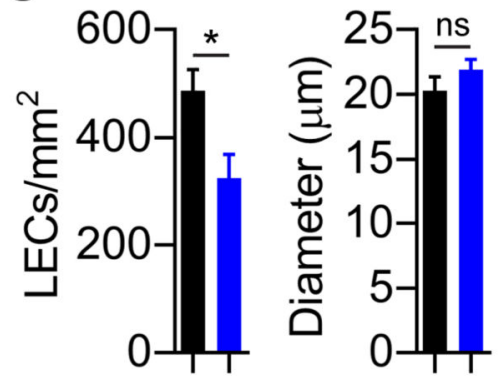

Ctrl

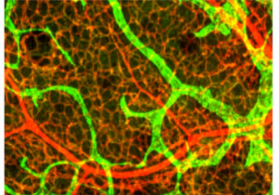

h

Figure 1. Inhibition of FGF signaling impairs lymphatic development.

a, Embryonic anterior dorsal skin (green area) was used to analyze lymphatic vessel development. b, Progressive ingrowth of lymphatic vessels (midline, dotted lines). c, Representative images of anterior dorsal skin from E15.5 embryos. Double-headed arrows indicate the distance between the leading fronts of the lymphatic vessels. Bottom panels (scale bars, $100 \mu \mathrm{m}$ ) are high-magnification images of boxed regions in upper panels (scale bars, $250 \mu \mathrm{m})$. d, e, Quantification of the distance between the leading fronts of lymphatic vessels (d; $n=3$ litters) and the number of lymphatic branch points per $\mathrm{mm}^{2}$ skin area (e; $n$ $=4$ embryos for control $\left(F g f r 1^{\text {flox/flox }} ; F_{g f f} 3^{+/-}\right) ; n=10$ embryos for

$\left.F g f r 1^{i \triangle L E C(B A C)} ; F g f r 3^{-/}\right)$. f, Representative images for VEGFR3 and PROX1 staining in the 
skin of E15.5 embryos. Scale bar, $150 \mu \mathrm{m}$. $\mathbf{g}, \mathbf{h}$, Quantification of the number of LECs per $\mathrm{mm}^{2}$ skin area (g) and lymphatic vessel diameter (h). $n=4$ embryos for control $\left(\right.$ Fgfr $\left.^{\text {flox/flox }} ; \mathrm{Fgfr}^{+/-}\right) ; n=10$ embryos for Fgfr $1^{i \Delta L E C(B A C)} ; \mathrm{Fgfr}^{-{ }^{--}}$. Data represent mean \pm s.e.m., ${ }^{*} P<0.05,{ }^{* *} P<0.01, \mathrm{~ns}=$ non-significant, calculated by unpaired t test $(\mathbf{d}, \mathbf{e}, \mathbf{h})$ and unpaired t test with Welch's correction $(\mathbf{g})$. 
a

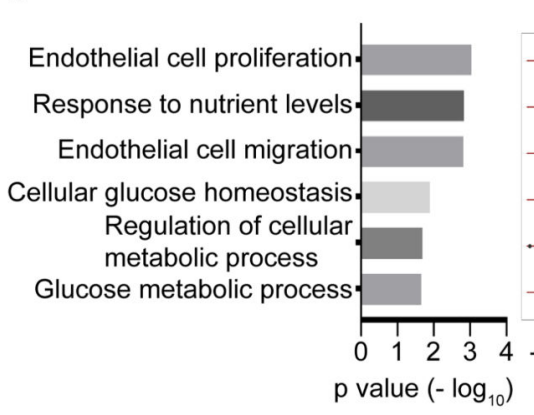

C

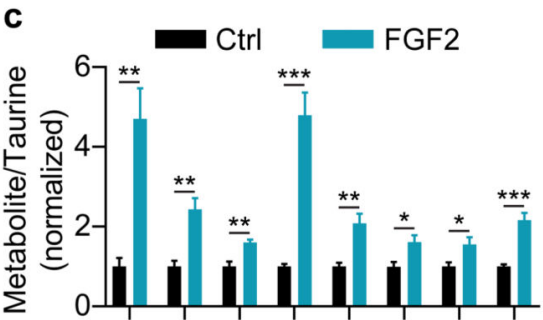

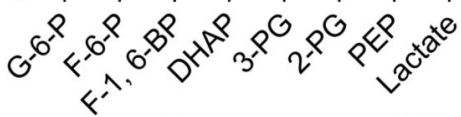

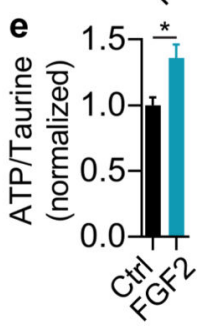

Ctrl FGFR1

i SIRNA SIRNA
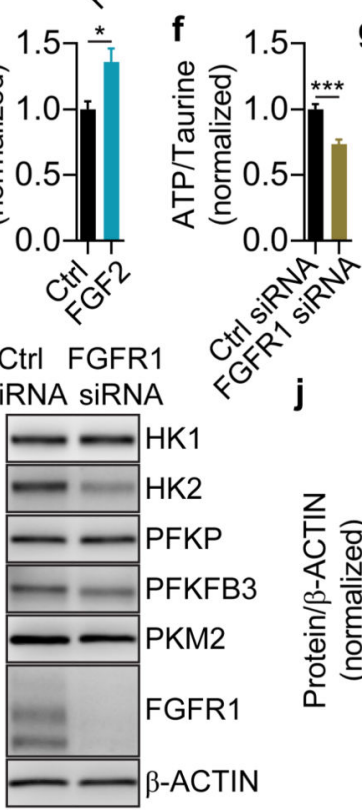

FGF2 FGFR1 SiRNA b
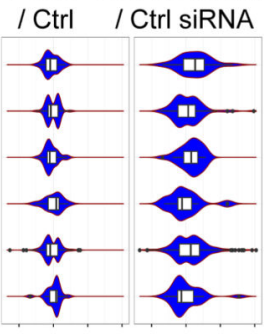

$\begin{array}{llllllll}-2 & 0 & 2 & 4 & -2 & 0 & 2 & 4\end{array}$

$\log _{2}$ (fold change)

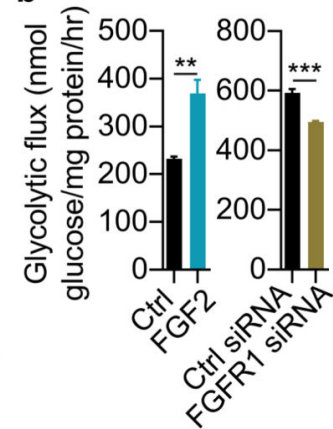

d

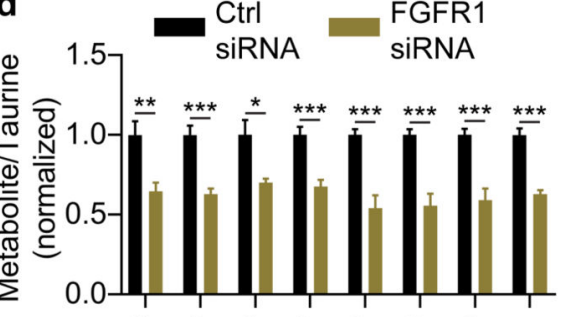

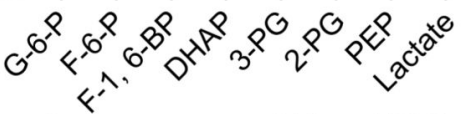

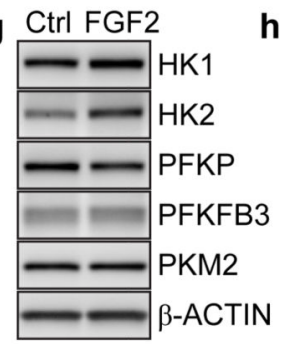

h
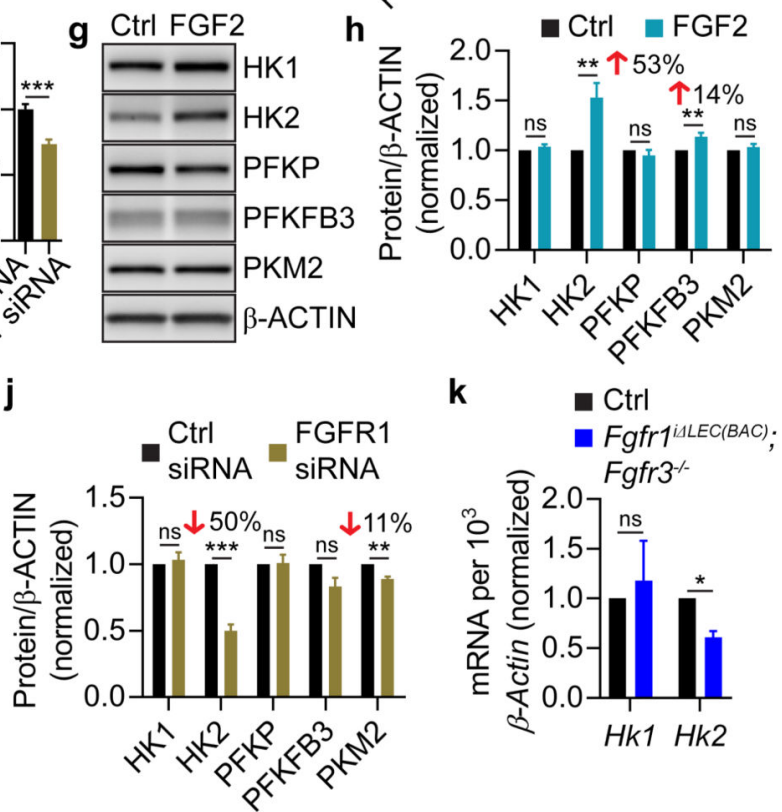

k

- Ctrl

- Fgfr 1 $^{\mathrm{ALEC}(\mathrm{BAC})}$;

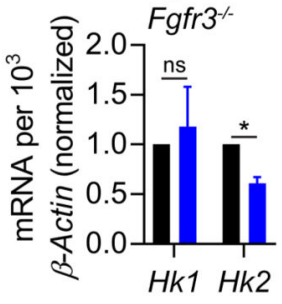

Figure 2. FGF signaling controls glycolysis and HK2 expression.

a, Enriched nested gene ontology (nGO) categories (left panel) in the FGF signalingregulated genes, identified by RNA-seq analysis of FGF2 and FGFR1 siRNA-treated HDLECs, and violin plots (right panel) showing the $\log _{2}$ fold change distributions of differentially expressed genes for each enriched nGO term. The width of violin plot indicates relative gene frequency at specific $\log _{2}$ fold change. $\mathbf{b}$, Left panel, measurement of glycolytic flux in control and FGF2-treated HDLECs $(n=4$ wells of samples for each condition, representative of 3 independent experiments). Right panel, glycolytic flux 
measurement in HDLECs transfected with control or FGFR1 siRNA ( $n=4$ wells of samples for each condition, representative of 2 independent experiments). c, d, Mass spectrometry measurement of glycolytic intermediates and lactate in control and FGF2- and FGFR1 siRNA-treated HDLECs ( $n=6$ wells of samples for each condition, representative of 2-3 independent experiments). e, f, Mass spectrometry measurement of ATP generation ( $n=6$ wells of samples for each condition, representative of 2 independent experiments). $\mathbf{g}, \mathbf{h}$, Western blot analysis (g) and densitometric quantification (h) of glycolytic enzyme expression in control or FGF2-treated HDLECs ( $n=6$ experiments). i, j, Immunoblot analysis (i) and densitometric quantification (j) of glycolytic enzyme expression in HDLECs treated with control siRNA or FGFR1 siRNA ( $n=3$ independent experiments). $\mathbf{k}$, qPCR analysis of $H k 1$ and $H k 2$ expression in dermal LECs isolated from E15.5 Fgfr ${ }^{i \Delta L E C(B A C)} ; F^{-1}{ }^{-/} 3^{-}$and control embryos with tamoxifen injection at E12.5 and E13.5 ( $n=2$ litters including 4 control and $2 \mathrm{Fgfr} 1^{i \Delta L E C(B A C)} ; \mathrm{Fgfr}^{-/}{ }^{-/}$embryos). Data represent mean \pm s.e.m., $* P<0.05, * * P<0.01, * * * P<0.001$, ns $=$ non-significant, calculated by unpaired $\mathrm{t}$ test $(\mathbf{b}-\mathbf{f}, \mathbf{h}, \mathbf{j}, \mathbf{k})$. For gel source data, see Supplementary Fig. 1. 


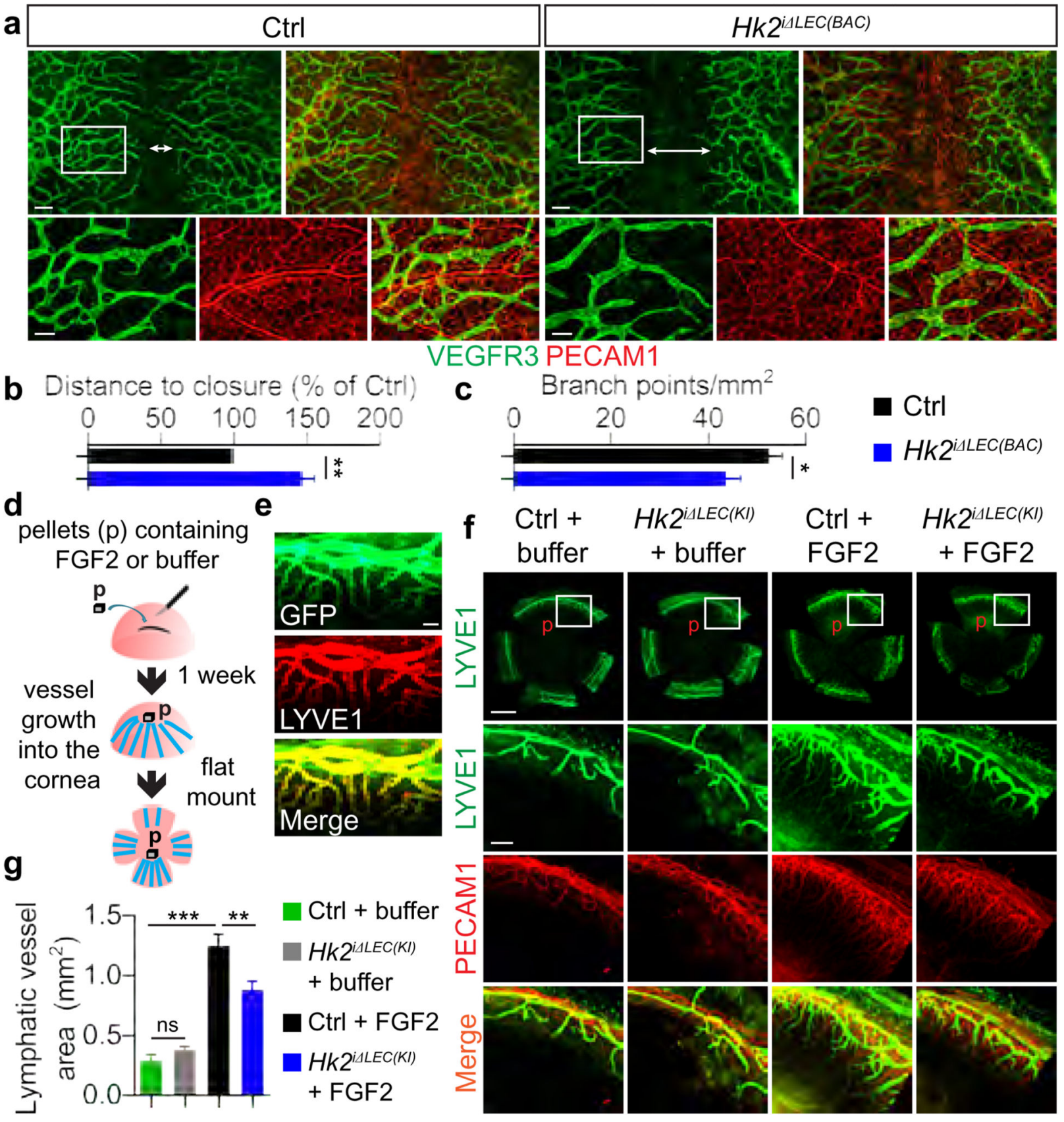

Figure 3. HK2 is essential for lymphangiogenesis.

a, Representative images of anterior dorsal skin stained with VEGFR3 and PECAM1 from E15.5 Hk $2^{i \Delta L E C(B A C)}$ and control embryos. Double-headed arrows indicate the distance between the two leading fronts of the lymphatic vessels. Bottom panels (scale bars, $100 \mu \mathrm{m}$ ) are high-magnification images of boxed regions in upper panels (scale bars, $250 \mu \mathrm{m}$ ). b, c, Quantification of the distance between the leading fronts of lymphatic vessels $(\mathbf{b} ; n=4$ litters) and the number of lymphatic branch points per $\mathrm{mm}^{2}$ skin area (c; $n=12$ embryos for control $\left(H k 2^{\text {flox/flox }}\right.$ or $\left.H k 2^{\text {flox/t }}\right) ; n=7$ embryos for $\left.H k 2^{i \Delta L E C(B A C)}\right)$. d, Cornea 
lymphangiogenesis model. e, Confocal images of FGF2-implanted cornea of Prox 1$\mathrm{CreER}^{T 2(K I)} ; m T m G$ reporter mice. Scale bar, $100 \mu \mathrm{m}$. f, Representative images of $H k 2^{i \Delta L E C(K I)}$ and control mouse corneas implanted with FGF2 or buffer containing pellets and stained for LYVE1 and PECAM1. Boxed regions in top-panel images (scale bar, 1000 $\mu \mathrm{m})$ are shown at high magnification in lower panels (scale bar, $200 \mu \mathrm{m}$ ). g, Quantification

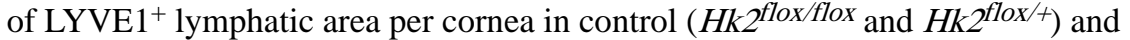
$H k 2^{i \Delta L E C(K I)}$ mice ( $n=21$ corneas for control + buffer; $n=5$ corneas for $H k 2^{i \Delta L E C(K I)}+$ buffer; $n=11$ corneas for control + FGF2; $n=14$ corneas for $H k 2^{i \Delta L E C(K I)}+$ FGF2). Data represent mean \pm s.e.m., $* P<0.05, * * P<0.01, * * * P<0.001$, ns $=$ non-significant, calculated by unpaired t test $(\mathbf{b}, \mathbf{c})$ and One-way ANOVA plus Sidak's multiple comparisons test $(\mathbf{g})$. 


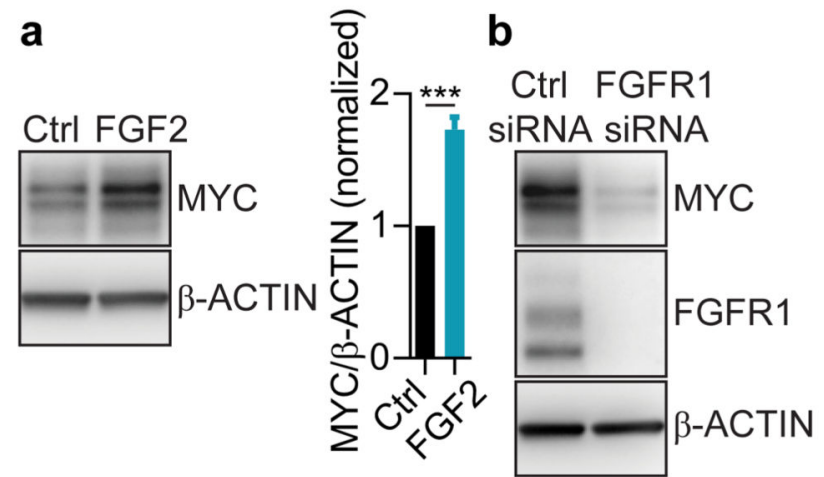

d
Ctrl MYC SIRNA siRNA $++\ldots+$ FGF2

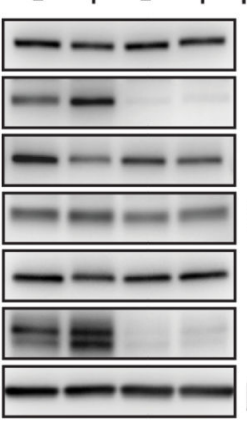

HK1

HK2

PFKP

PFKFB3

PKM2

MYC

B-ACTIN

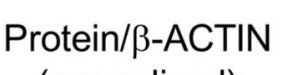

(normalized) e

Ctrl FGFR1 SIRNA SiRNA

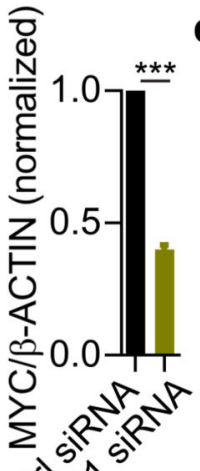

0

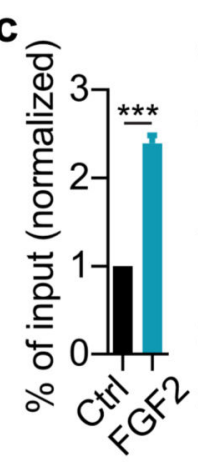

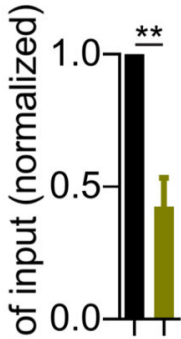

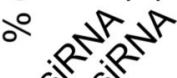<smiles>C1#CC1</smiles><smiles>C1=[Ge]=C[GeH]=1</smiles>

\section{Protein/ $\beta$-ACTIN} (normalized)

$\begin{array}{lllll}0.0 & 0.5 & 1.0 & 1.5 & \mathrm{Ad}-\overline{\mathrm{Ctrl}} \overline{\mathrm{Ctrl} M Y C}\end{array}$

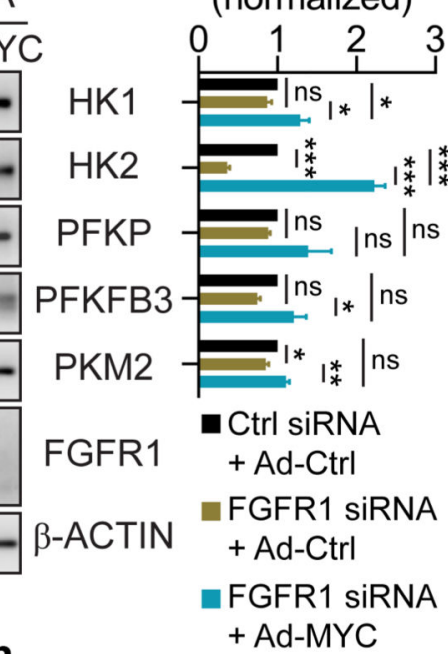

VEGFR3 PROX1
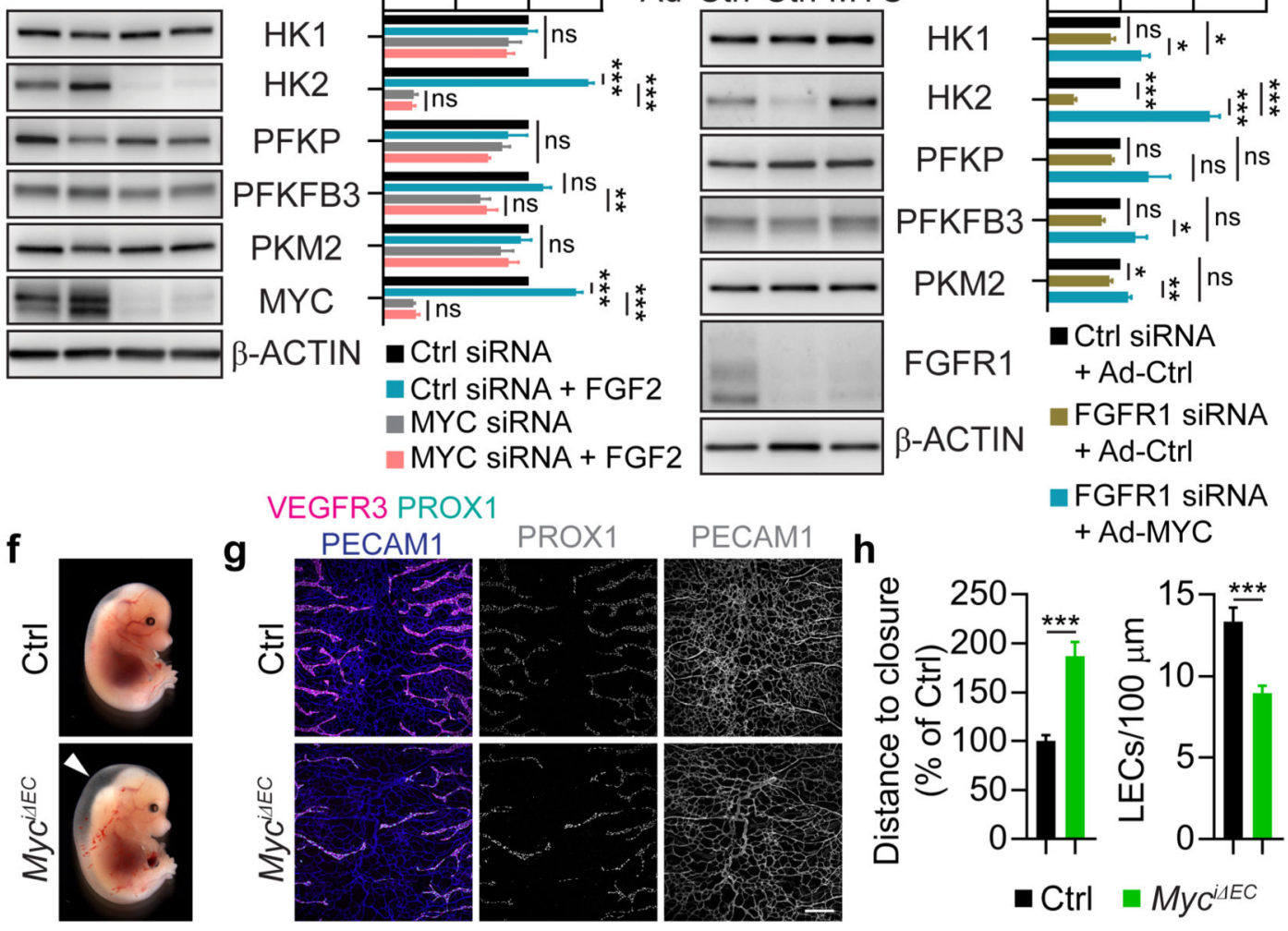

Figure 4. MYC mediates FGF regulation of HK2 expression and is critical for lymphatic development.

a, MYC protein expression in control or FGF2-treated HDLECs $(n=4$ replicates from 2 independent experiments). b, MYC protein expression in HDLECs treated with control siRNA or FGFR 1 siRNA ( $n=4$ replicates from 2 independent experiments). c, ChIP-qPCR analysis of MYC binding to the regulatory region of $H K 2$ gene in HDLECs under different treatments as indicated ( $n=3$ independent experiments). d, MYC and glycolytic enzyme protein levels in siRNA-transfected HDLECs with or without FGF2 treatment $(n=3$ 
experiments). e, Glycolytic enzyme and FGFR1 protein levels in siRNA-transfected HDLECs treated with control or MYC adenovirus ( $n=2-3$ replicates from 2 experiments). f, Bright-field images of E15.5 Myc $c^{i \Delta E C}$ and control ( $\left.M y c^{f l o x} / f l o x\right)$ embryos. Arrowhead denotes area with lymphedema. g, Confocal images of anterior dorsal skin with VEGFR3, PROX1 and PECAM1 staining from E15.5 Myc $\mathrm{c}^{\mathrm{ALEC}}$ and control embryos. Scale bar, 250 $\mu \mathrm{m}$. $\mathbf{h}$, Quantification of the distance between the leading fronts of ingrowing lymphatics (left panel; $n=10$ embryos for control (Myclox/flox $) ; n=5$ embryos for $M y c^{i \Delta E C}$ ) and the number of LECs (PROX1 staining) per 100- $\mu \mathrm{m}$ length of lymphatic vessels (right panel; $n=$ 6 embryos for control (Myctlox/flox $) ; n=9$ embryos for $M y c^{i \Delta E C}$ ). Data represent mean \pm s.e.m., $* P<0.05, * * P<0.01$, *** $P<0.001$, ns $=$ non-significant, calculated by unpaired $\mathrm{t}$ test $(\mathbf{a}-\mathbf{c}, \mathbf{h})$ and One-way ANOVA plus Sidak's (d) or Tukey's (e) multiple comparisons test. For gel source data, see Supplementary Fig. 1. 